\title{
An international ISHLT/ATS/ERS clinical practice guideline: diagnosis and management of bronchiolitis obliterans syndrome
}

\author{
Keith C. Meyer ${ }^{1}$, Ganesh Raghu², Geert M. Verleden ${ }^{3}$, Paul A. Corris', \\ Paul Aurora ${ }^{5}$, Kevin C. Wilson ${ }^{6}$, Jan Brozek ${ }^{7}$, Allan R. Glanville ${ }^{8}$ and \\ the ISHLT/ATS/ERS BOS Task Force Committee ${ }^{9}$
}

\begin{abstract}
Affiliations: 'School of Medicine and Public Health, University of Wisconsin-Madison, Madison, WI, USA. ${ }^{2}$ School of Medicine, University of Washington, Seattle, WA, USA. ${ }^{3}$ University of Leuven, Leuven, Belgium. ${ }^{4}$ Freeman Hospital, Newcastle upon Tyne, UK. ${ }^{5}$ Great Ormond Street Hospital for Children, London, UK. ${ }^{6}$ Boston University Medical Center, Boston, MA, USA. ${ }^{7}$ McMaster University, Hamilton, ON, Canada. ${ }^{8}$ The Lung Transplant Unit, St Vincents Hospital, Sydney, Australia. ${ }^{9} \mathrm{~A}$ full list of the ISHLT/ATS/ERS BOS Task Force Committee members and their affiliations can be found in the Acknowledgements section.
\end{abstract}

Correspondence: Keith C. Meyer, University of Wisconsin Lung Transplant and Advanced Pulmonary Disease Program, Section of Allergy, Pulmonary and Critical Care Medicine, Department of Medicine, University of Wisconsin, Madison, WI, USA. E-mail: kcmamedicine.wisc.edu

ABSTRACT Bronchiolitis obliterans syndrome (BOS) is a major complication of lung transplantation that is associated with poor survival. The International Society for Heart and Lung Transplantation, American Thoracic Society, and European Respiratory Society convened a committee of international experts to describe and/or provide recommendations for 1) the definition of BOS, 2) the risk factors for developing $\mathrm{BOS}, 3)$ the diagnosis of BOS, and 4) the management and prevention of BOS.

A pragmatic evidence synthesis was performed to identify all unique citations related to BOS published from 1980 through to March, 2013. The expert committee discussed the available research evidence upon which the updated definition of BOS, identified risk factors and recommendations are based. The committee followed the GRADE (Grading of Recommendation, Assessment, Development and Evaluation) approach to develop specific clinical recommendations.

The term BOS should be used to describe a delayed allograft dysfunction with persistent decline in forced expiratory volume in $1 \mathrm{~s}$ that is not caused by other known and potentially reversible causes of posttransplant loss of lung function. The committee formulated specific recommendations about the use of systemic corticosteroids, cyclosporine, tacrolimus, azithromycin and about re-transplantation in patients with suspected and confirmed BOS.

The diagnosis of BOS requires the careful exclusion of other post-transplant complications that can cause delayed lung allograft dysfunction, and several risk factors have been identified that have a significant association with the onset of BOS. Currently available therapies have not been proven to result in significant benefit in the prevention or treatment of BOS. Adequately designed and executed randomised controlled trials that properly measure and report all patient-important outcomes are needed to identify optimal therapies for established BOS and effective strategies for its prevention.

@ERSpublications

Diagnosis of BOS requires careful exclusion of other complications that can cause delayed lung allograft dysfunction http://ow.ly/AZmbr

This article has supplementary material available from erj.ersjournals.com

Received: June 122014 | Accepted after revision: Aug 262014 | First published online: Oct 302014

Conflict of interest: Disclosures can be found alongside the online version of this article at erj.ersjournals.com

Copyright @ERS 2014 


\section{Executive summary}

Many lung transplant recipients develop delayed allograft dysfunction that has been traditionally referred to as bronchiolitis obliterans syndrome (BOS), which is thought to be caused by inflammation, destruction and fibrosis of small airways in the lung allograft that leads to obliterative bronchiolitis (OB). Because a definitive diagnosis of $\mathrm{OB}$ is difficult to make without a surgical lung biopsy, a decrease in the forced expiratory volume in $1 \mathrm{~s}(\mathrm{FEV} 1)$ has been used as a surrogate marker to identify patients who develop a syndrome of significant and persistent loss of lung allograft function with onset three or more months following transplantation. However, it is now recognised that numerous factors apart from OB can lead to a delayed onset, significant decline in lung function, and these causes of delayed onset graft dysfunction must be carefully excluded when a diagnosis of BOS is made. In general, BOS responds poorly to therapeutic interventions but may stabilise, and some patients may have a significant improvement in FEV1 with certain therapies.

A comprehensive review of the literature on lung transplantation and BOS allowed the committee to reach a number of conclusions, which are given in table 1. In addition, committee members used a systematic approach to formulate a number of specific evidence-based recommendations for the prevention and management of BOS, which are given in table 2. Evidence tables are provided in the online supplementary material. It is our hope that this guideline will promote an understanding of the current approach to the evaluation and management of lung transplant recipients who develop delayed allograft dysfunction, as well as stimulate additional research that will provide higher quality evidence upon which future guidelines may be based.

\section{Scope and purpose}

The purpose of this document is to revise the definition of BOS, discuss the risk factors for the development of BOS, and provide guidance about the management of patients with suspected or confirmed BOS. The target audience for these guidelines are specialists in respiratory medicine managing adults and children who have received lung transplants. Other specialists in respiratory medicine may also benefit from these guidelines.

\section{Introduction}

OB, first described in recipients of heart-lung transplants in 1984 [1], is recognised as a major cause of lung allograft dysfunction following lung transplantation [2-5]. Post-transplant $O B$ is characterised by progressive obliteration of small airways (fig. 1) that is typically accompanied by a persistent decline in spirometric measures of lung function, a spirometric pattern that is usually obstructive, and an essentially clear chest radiograph [4]. However, OB is difficult to detect via transbronchial lung biopsy and cannot be confidently diagnosed via noninvasive testing [6-10]. Therefore, previously published consensus statements have designated a persistent decline in FEV1 to $\leqslant 80 \%$ of baseline post-transplant FEV 1 that is present for a minimum of 3 weeks (in the absence of confounding conditions) as a surrogate marker of probable OB, and such FEV1 decline has been termed BOS $[4,5]$.

The BOS classification scheme, adopted in 1993 [4], provided a staging system based on the severity of lung function decline after transplant and has been used for clinical decision-making and research purposes. This staging system was most recently modified in 2002 (table 3) [5]. Baseline values for FEV1 and forced expiratory flow at $25-75 \%$ of forced vital capacity (FEF25-75\%) are defined as the average of the two highest values for each measurement that were obtained at least 3 weeks apart post-transplant without the administration of a bronchodilator. To help distinguish BOS from acute and/or subacute complications of lung transplantation, and taking into account the time needed to establish both a baseline FEV1 and a decline in FEV1 ascertained by two FEV1 measurements performed 3 weeks apart, by definition, three or more months are required to have elapsed from the time of transplantation in order for the diagnosis of BOS to be made $[4,5]$. In addition, it has become clear that lung function decline consistent with a diagnosis of BOS can stabilise in some patients and not lead to sustained, progressive deterioration in allograft function and graft loss. Because of concern that setting the cut-off value for FEV1 at $80 \%$ of the best post-transplant value may be insensitive to early decline in allograft function due to early $\mathrm{OB}$, BOS Grade 0 -p ( $\geqslant 10 \%$ but $<20 \%$ decline in FEV1 and/or $\geqslant 25 \%$ decline in FEF25-75\%) was added to the staging system to signify "potential BOS" [5].

BOS affects $50 \%$ or more of recipients who survive beyond 5 years and accounts for a considerable proportion of cases of lung allograft loss and recipient death beyond 3 months post-transplant. It is the leading cause of death for recipients who survive beyond 1 year post-transplant $[2,3]$, and it is widely perceived as the physiological surrogate of immunologically-mediated phenomena due to many observations that include its association with acute cellular rejection [11], the tendency of recipients who develop BOS to have greater degrees of human leukocyte antigen (HLA) mismatch [12], and accumulating 


\section{TABLE 1 Conclusions}

1) The terms BOS and CLAD should not be considered interchangeable or synonymous. Both are clinical terms that describe clinical syndromes. CLAD needs a precise definition, which has not yet been determined.

2) The term BOS should be retained to denote allograft dysfunction with delayed onset and persistent decline in FEV1 (which is usually accompanied by evidence of airflow obstruction) that is not caused by other causes (some of which may be potentially reversible) of posttransplant loss of function.

3) The timing of BOS onset and its subsequent course provide prognostic information and may be linked to different pathophysiological mechanisms.

4) The identification and detailed definition of BOS phenotypes that correlate with prognosis and response to therapy may be useful in understanding the natural course of BOS and the development of more targeted treatment modalities.

5) The following potential risk factors are associated with BOS:

a) Primary graft dysfunction (PGD)

b) AR including Minimal Grade A1 and higher AR grades

c) Lymphocytic bronchiolitis (LB) or Grade B rejection

d) Antibody-mediated rejection (AMR)

e) Gastro-oesophageal reflux (GOR) (acid and non-acid)

f) Cytomegalovirus (CMV) pneumonitis

g) Symptomatic community-acquired respiratory virus (CARV) infection

h) Colonisation and infection of the lung by Pseudomonas aeruginosa

i) Aspergillus colonisation or fungal pneumonitis

j) Autoimmune sensitisation to collagen $V$

k) Increased BAL neutrophils on BAL differential cell count

6) BOS is generally suspected at an early stage when the FEV 1 is $\leqslant 90 \%$ of baseline (i.e. BOS Grade 0 -p) and/or the FEF $25-75 \%$ is $\leqslant 75 \%$ of baseline in both bilateral and single lung transplant recipients.

7) In most transplant centres, lung transplant recipients (including asymptomatic patients) receive sustained follow-up including routine clinical evaluation, spirometry (both in the clinic and in remote in-home settings), and other methods for monitoring allograft status (such as fibre-optic bronchoscopy, as appropriate). Such monitoring is generally sustained beyond the first 6-12 months following transplantation.

8) When lung transplant recipients who have been clinically stable develop a decline in lung function, prompt clinical evaluation is usually performed to identify the likely cause.

9) Routine postero-anterior and lateral chest radiographs are neither sensitive nor specific for diagnosing BOS.

10) The findings of air trapping with expiratory views and/or mosaic attenuation patterns on HRCT imaging of the thorax support the presence of BOS, but lack sensitivity and specificity.

11) Thoracic imaging assists in making a diagnosis of BOS by ruling out other causes of allograft function decline.

12) Surveillance bronchoscopy can safely evaluate the lung allograft for occult abnormalities, although a beneficial effect on recipient survival and prevention of BOS has not been clearly demonstrated. In most transplant centres, surveillance bronchoscopy is routinely offered to lung recipients to potentially allow early detection of occult chronic lung allograft dysfunction and/or the presence of occult infection.

13) Although bronchoscopy has poor sensitivity for the diagnosis of $O B$, bronchoscopy is frequently used to evaluate the lung allograft when evidence of clinical dysfunction is identified.

14) The presence of BAL neutrophilia suggests that $O B$ may be occurring in the lung allograft and that the allograft is at increased risk for progression to BOS; infection is a confounder and may be the cause of BAL neutrophilia, although infection and OB/BOS may coexist in the allograft.

15) The presence of donor-specific antibody (DSA) suggests AMR when detected in the context of a delayed allograft functional decline.

16) For lung transplant recipients who develop BOS and have evidence of allograft infection, aggressive measures to control and eradicate infection are routine.

17) Within the various classes of commonly used immunosuppressive agents in lung transplant recipients, there is no definitive evidence of superiority of one drug or drug combination for prevention of BOS.

18) Single-centre studies suggest that some less commonly used immunosuppressive agents (i.e. sirolimus, alemtuzumab and anti-thymocyte globulin) may improve outcomes in patients with BOS.

19) Extracorporeal photopheresis (ECPP) and total lymphoid irradiation (TLI) are therapies that some institutions consider for selected patients with progressive BOS.

BOS: bronchiolitis obliterans syndrome; CLAD: chronic lung allograft dysfunction; FEV1: forced expiratory volume in 1 s; AR: acute cellular rejection; BAL: bronchoalveolar lavage; FEF25-75\%: forced expiratory flow at 25-75\% of forced vital capacity; HRCT: high-resolution computed tomography; OB: obliterative bronchiolitis.

evidence of the involvement of autoimmune pathways [13]. Furthermore, there are striking similarities to $\mathrm{OB}$ that can occur in allogeneic bone marrow or stem cell transplant recipients as well as in patients with connective tissue diseases, which are also perceived as alloimmune or autoimmune disorders, respectively. Therefore, BOS is frequently equated with the term chronic rejection. However, various interventions, including intensified immunosuppression, may have little or no effect on progressive loss of allograft 


\section{TABLE 2 Recommendations}

1) For lung transplant recipients who have non-minimal acute cellular rejection (Grade $\geqslant 2$ ) or lymphocytic bronchitis on transbronchial lung biopsy specimens, we suggest augmented immunosuppression with a course of systemic steroids to prevent the development of BOS (conditional recommendation, very low quality of evidence).

Values and preferences: this recommendation places a high value on preventing a life-threatening complication of lung transplantation and a lower value on avoiding short-term adverse effects.

Remarks: a typical course of systemic corticosteroids used to augment immunosuppression in adult recipients is intravenous methylprednisolone $1000 \mathrm{mg}$ daily for 3 days (many centres use $10-15 \mathrm{mg} \cdot \mathrm{kg}^{-1}$ per day for smaller patients).

2) For lung transplant recipients who have clinically significant minimal acute cellular rejection (Grade A1) on transbronchial lung biopsy specimens, we suggest augmented immunosuppression with a course of systemic steroids to prevent the development of BOS if the finding of grade $\mathrm{A} 1$ acute cellular rejection is perceived to be clinically significant (conditional recommendation, very low quality of evidence).

Values and preferences: this recommendation places a high value on preventing a life-threatening complication of lung transplantation and a lower value on avoiding short-term side-effects.

Remarks: we consider Grade A1 acute cellular rejection to be clinically significant if it is associated with clinical findings, such as symptoms (e.g. dyspnoea, fatigue or new-onset cough) or objective measurements (e.g. decline in FEV1 or oxyhaemoglobin desaturation with ambulation), that suggest the presence of allograft dysfunction. A typical course of systemic steroids used to augment immunosuppression in adult recipients is intravenous methylprednisolone $1000 \mathrm{mg}$ daily for 3 days (many centres use $10-15 \mathrm{mg} \cdot \mathrm{kg}^{-1}$ per day for smaller patients).

3) For lung transplant recipients who develop a decline in FEV1 consistent with the onset of BOS, we suggest that clinicians do not use longterm, high-dose corticosteroids (conditional recommendation, very low quality of evidence).

Values and preferences: this recommendation places a high value on avoiding harmful effects due to ineffective therapies.

Remarks: we define sustained administration of high-dose corticosteroid as $\geqslant 30 \mathrm{mg} \cdot \mathrm{day}^{-1}$ of prednisone or an equivalent formulation

4) For lung transplant recipients who develop BOS while receiving chronic immunosuppression with a regimen that includes cyclosporine, we suggest switching the cyclosporine to tacrolimus (conditional recommendation, very low quality of evidence).

Values and preferences: this recommendation places a higher value on mitigation of lung function decline and a lower value on avoiding nephrotoxicity and hyperglycaemia.

Remarks: the conversion of cyclosporine to tacrolimus is generally performed by stopping cyclosporine and initiating tacrolimus while transiently increasing maintenance corticosteroid dosing until tacrolimus blood levels are ascertained to have reached the desired target range. The target for therapeutic trough blood levels of tacrolimus is generally considered to range from 5 to $15 \mathrm{ng} \cdot \mathrm{mL}^{-1}$ for patients who are $\geqslant 18$ years of age once a steady state has been attained.

5) For lung transplant recipients who develop a decline in FEV1 consistent with the onset of BOS, we suggest a trial of azithromycin (conditional recommendation, very low quality of evidence).

Values and preferences: this recommendation places a high value on preventing lung function deterioration and possibly reducing mortality and a lower value on avoiding adverse effects.

Remarks: azithromycin is generally administered orally at $250 \mathrm{mg}$ per day for 5 days and then at $250 \mathrm{mg}$ three times per week. We define a trial of azithromycin as treating continuously with azithromycin for a minimum of 3 months. Additionally, it is unclear 1) whether azithromycin should be continued long-term if a beneficial response is observed, or 2) whether it should be discontinued if lung function does not show improvement during follow-up clinical evaluation.

6) For lung transplant recipients who develop a decline in FEV1 consistent with the onset of BOS and have confirmed GOR, we suggest referral to an experienced surgeon to be evaluated for potential fundoplication of the gastro-oesophageal junction (conditional recommendation, very low quality of evidence).

Values and preferences: this recommendation places a high value on reducing the risk of lung function deterioration, and possibly mortality, and a lower value on avoiding surgical complications.

Remarks: Nissen fundoplication has been more extensively studied than Toupet fundoplication; however, we have no reason to believe that one is superior to the other and feel that the choice of the surgical technique should remain at the surgeon's discretion.

7) For lung transplant recipients who have developed end-stage BOS refractory to other therapies, we recommend referral to a transplant surgeon to be evaluated for re-transplantation (conditional recommendation, very low quality of evidence).

Values and preferences: this recommendation places a high value on avoiding surgical complications (e.g. mortality), recurrent BOS and resource utilisation.

Remarks: the selection process for re-transplantation is the same as that used for first-time lung transplantation.

BOS: bronchiolitis obliterans syndrome; FEV1: forced expiratory volume in 1 s; GOR: gastro-oesophageal reflux.

function in patients with BOS. Additionally, many nonimmune mechanisms have also been implicated or suggested to play a role in BOS pathogenesis. These include airway injury due to primary graft dysfunction (PGD), gastro-oesophageal reflux (GOR), various infections, and airway ischaemia due to disruption of the bronchial circulation [14-16]. These "non-immune" factors may promote tissue damage and inflammation that in turn initiates and intensifies an alloimmune recipient response. Established OB displays variable evidence of inflammation, alloimmune reactions, autoimmunity, and fibroproliferation with airway obliteration that leads to allograft airway remodelling and loss of function [14-16]. OB may represent a final common end-point for a variety of forms of allograft injury. 

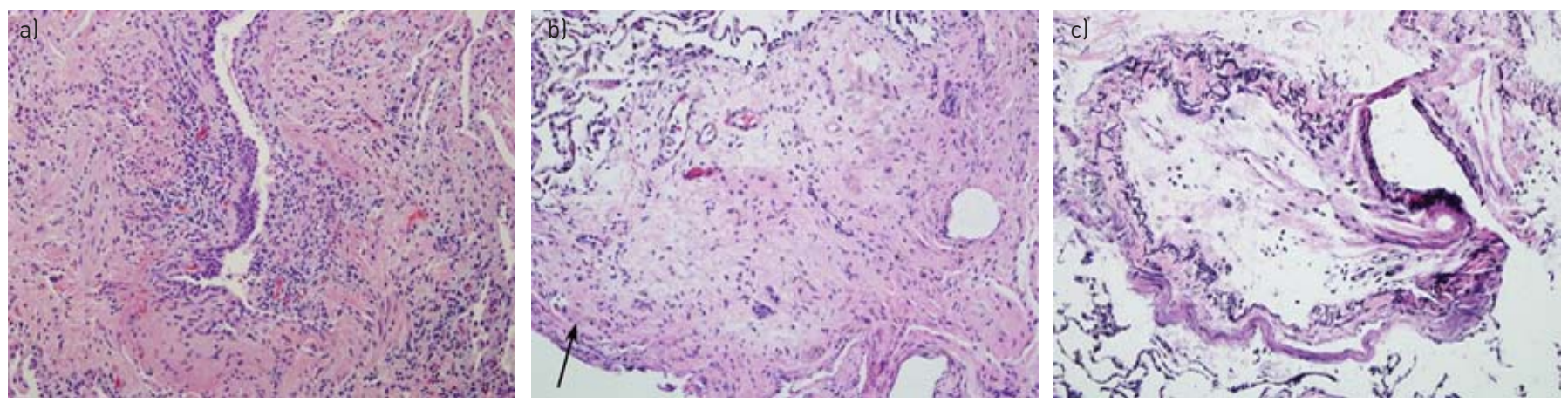

FIGURE 1 a) Bronchiolitis obliterans in a surgical lung biopsy with partial luminal compromise accompanied by mild chronic inflammation in the wall and focal ulceration of the mucosa. b) Bronchiolitis obliterans on a transbronchial biopsy with complete luminal obliteration. Scant bronchiolar muscle (arrow) helps to identify the scarred structure as residual airway (haematoxylin and eosin stain). c) An elastic tissue stain from a slightly deeper section of the same bronchiole (shown in b) highlights the residual elastica present. In contrast to the accompanying artery on the right, there is only one elastic lamella in the bronchiolar wall. Original magnification: $100 \times$.

Because BOS is clinically defined by a persistent decline in lung function, post-transplant decline in FEV1 may be incorrectly perceived as exclusively due to OB. It has been increasingly recognised that other allograft disorders can occur in the chronic post-transplant setting (table 4). Some of these entities may cause allograft dysfunction that may not be reversible and yet meet the spirometric criteria for the diagnosis of BOS, as many of these entities may also lead to a sustained decline in FEV1. The situation can be further complicated by the simultaneous existence of other pathophysiological entities (infection, various forms of rejection and diffuse alveolar damage) when $\mathrm{OB}$ is also present in the allograft.

This International Society for Heart and Lung Transplantation (ISHLT)/American Thoracic Society (ATS)/ European Respiratory Society (ERS) clinical practice guideline provides a comprehensive, conceptually balanced and evidence-based perspective that examines the concepts pertaining to the diagnosis and management of BOS that have appeared in the medical literature since this syndrome was first described. It is intended to provide guidance in management whenever possible, and to identify gaps in knowledge and issues that need to be addressed via additional basic and clinical research. It should be recognised that the vast majority of patients described in the published literature have been adults, and some recommendations may not have as firm a basis when applied to paediatric lung transplant recipients.

\section{How to use these guidelines}

The ISHLT/ATS/ERS guidelines about the management of BOS are not intended to impose a standard of care. They provide the basis for rational decisions in the management of patients with suspected or

TABLE 3 Grading (staging) of bronchiolitis obliterans syndrome (BOS) ${ }^{\#}$

BOS Grade

Spirometry $\%$ of baseline

1993 Classification

$\mathrm{FEV} 1 \geqslant 80 \%$

Not included

FEV1 $66-80 \%$

FEV1 $51-65 \%$

FEV $1 \leqslant 50 \%$

\section{Classification}

$\mathrm{FEV}_{1}>90 \%$ and $\mathrm{FEF}_{25}-75 \%>75 \%$

FEV1 $81-90 \%$ and/or FEF $25-75 \% \leqslant 75 \%$

FEV1 $66-80 \%$

FEV $151-65 \%$

FEV $1 \leqslant 50 \%$

FEV1: forced expiratory volume in $1 \mathrm{~s}$; FEF25-75\%: forced expiratory flow at 25-75\% of forced vital capacity. \#: Other causes of lung function decline must be excluded le.g. acute rejection, infection, native lung problems for single lung recipients, excessive recipient weight gain, anastomotic dysfunction, respiratory muscle dysfunction, effusion, or technical problems such as erroneous measurements due to device dysfunction); $\tau^{\prime}$ : baseline is defined as the average of the two best FEV1 (or FEF25-75\%) values ( $\geqslant 3$ weeks apart) following functional recovery and stabilisation post-lung transplantation; ${ }^{+}$: in Grade (stage) 0-p the " $p$ " denotes "probable" early BOS and is used to indicate 10-20\% decline in baseline FEV1 that is likely due to an early stage of BOS that does not meet criteria for BOS Grade 1. 


\section{TABLE 4 Differential diagnosis of delayed post-transplant lung function decline}

1) Bronchiolitis obliterans syndrome (BOS)

2) Non-BOS alloinflammatory processes

a) Acute cellular rejection

b) Lymphocytic bronchiolitis

c) Antibody-mediated rejection (humoral, vascular)

\section{3) Restrictive allograft syndrome}

4) Inflammatory complications of the lung allograft

a) Pleuro-parenchymal inflammation

i) Bronchiolitis obliterans organising pneumonia (BOOP)

ii) Fibrinoid and organising pneumonia (FOP)

b) Chronic inflammation of airways

i) Large airways (bronchiectasis" ${ }^{\#}$, bronchomalacia)

ii) Bronchioles (follicular or exudative bronchiolitis)

c) Chronic pleural inflammation

d) Chronic vascular rejection

5) Infection

6) Surgical removal of lung tissue

7) Mechanical abnormality

a) Airway dysfunction

i) Anastomotic stricture/stenosis

ii) Bronchomalacia (allograft, native airway in SLT)

b) Allograft compression

i) Weight gain

ii) Abdominal distention

iii) Hyperinflation of native lung in SLT for emphysema

iv) Pleural complications

1. Pneumothorax

2. Pleural effusion

3. Pleural fibrosis

4. Bronchopleural fistula

c) Impaired graft inflation

i) Pain (vertebral fracture, fracture of ribs and/or sternum)

ii) Ventilatory compromise

1. Diaphragmatic dysfunction or paralysis

2. Chest wall myopathy

iii) Other (cerebrovascular accident, Parkinson's disease, etc.)

d) Drug reaction (e.g. sirolimus, everolimus and amiodarone)

e) Pulmonary oedema

f) Malignancy (PTLD or other)

8) Vascular obstruction

a) Allograft anastomotic large vessel strictures

b) Thromboembolic disease

c) Tumour emboli

\section{9) Allograft parenchymal abnormalities}

a) Transplant indication disease recurrence

i) Interstitial diseases (e.g. sarcoidosis, PLCH and LAM)

ii) Other (e.g. veno-occlusive disease, connective tissue disorders)

b) Diffuse alveolar damage

c) Organising pneumonia

10) Ageing

SLT: single lung transplant; PTLD: post-transplant lymphproliferative disease; PLCH: pulmonary Langerhans' cell histiocytosis; LAM: lymphangioleiomyomatosis. \#: Bronchiectasis may be a manifestation of obliterative bronchiolitis/BOS.

confirmed BOS. Clinicians, patients, third-party payers, institutional review committees, other stakeholders or the courts should never view these recommendations as dictates. No guidelines and recommendations can take into account all of the often compelling, unique individual clinical circumstances. Therefore, no one charged with evaluating clinicians' actions should attempt to apply the recommendations from these guidelines by rote or in a blanket fashion. 
Statements about the underlying values and preferences as well as the qualifying remarks accompanying each recommendation are integral parts and serve to facilitate more accurate interpretation. They should never be omitted when quoting or translating recommendations from these guidelines.

\section{Methods}

An ISHLT, ATS and ERS sponsored ad hoc committee held preliminary meetings in April and May of 2008 to begin the process of identifying and prioritising topics to be covered in this guideline. The chairs were approved by the three societies. Panel members were identified as leaders in the field of lung transplantation and were selected from established transplant centres worldwide, by the chairs, to review the existing literature and to answer clinical questions based upon the published evidence or, when such evidence was lacking, to provide guidance based upon the observations in their clinical practice. All members of the committee disclosed potential conflicts of interest, which were vetted according to the policies of the ISHLT, ATS and ERS. Each member of the committee was involved in developing the conclusions and recommendations provided by this document.

A comprehensive literature search was performed by a medical librarian. The PubMed interface was used to search MEDLINE for relevant publications (original articles and systematic reviews) in the English language from 1980 through to 2009. The search was updated twice in 2012 and in March, 2013. The search terms included "lung transplantation", "bronchiolitis obliterans syndrome" and terms specific to management options considered in the clinical questions. A total of 10031 manuscripts were identified using the electronic searches. Relevant publications were selected by committee members using pre-specified inclusion criteria, and the bibliographies of selected articles were reviewed to identify additional articles. The pragmatic evidence synthesis was primarily qualitative, rather than quantitative (i.e. few data could be pooled via meta-analysis). The methods used for this guideline are summarised in table 5.

Members of the committee were provided with the entire collection of compiled documents and subcommittees were formed to address specific topics. Each subcommittee reviewed, appraised and summarised the relevant evidence. The GRADE (Grading of Recommendation, Assessment, Development and Evaluation) approach was used to appraise the quality of the body of evidence supporting each recommendation. Clinical questions related to treatment versus no treatment, one treatment versus an alternative treatment, or which populations to treat were answered with recommendations that were formulated and graded using the GRADE approach (table 6) [17]. Disagreements were resolved by discussion and consensus. The final recommendations and grades were reviewed by the entire committee and approved in September, 2013. In contrast to the systematically developed recommendations, other committee conclusions were based upon the literature appraisal and committee deliberations.

A strong recommendation was made if the committee felt confident of the balance between desirable and undesirable consequences. A conditional recommendation was made if the committee felt less confident of

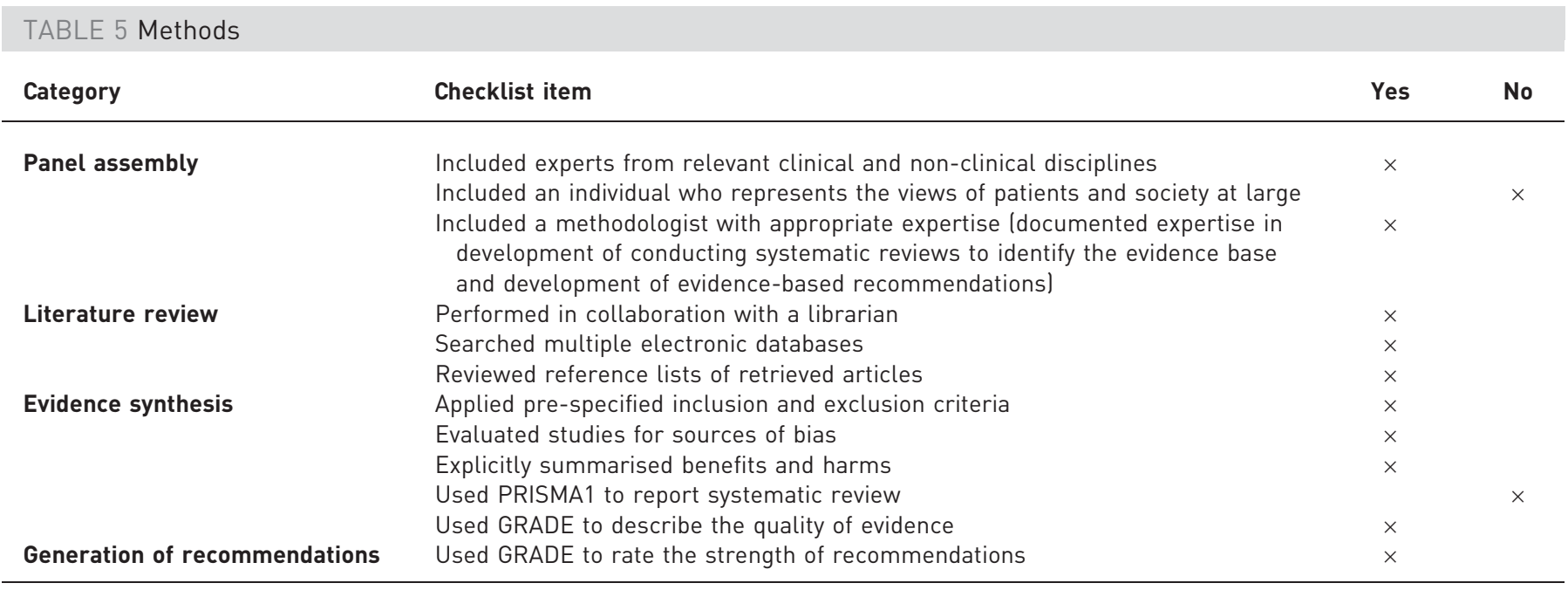

PRISMA: Preferred Reporting Items for Systematic Reviews and Meta-Analyses; GRADE: Grading of Recommendation, Assessment, Development and Evaluation. 
the balance between desirable and undesirable consequences. Factors that influence the strength of recommendations include the estimates of effect for desirable and undesirable outcomes of interest, confidence in these estimates of effects, estimates of values and preferences, and resource use. In any case the appropriate course of action depends upon the clinical context. The committees' judgments about the underlying values and preferences of well-informed patients were based upon the committee members' clinical experience. Evidence tables summarising the relevant literature for each recommendation are provided in the online supplementary material.

The committee identified very few experimental studies of the management of BOS. Available data are very limited owing to the small number of subjects. Thus, most of the recommendations are based upon observational studies with or without a control group and the clinical experience of the committee members (i.e. unsystematic clinical observations from their clinical practices).

\section{Terminology used for BOS}

Several confounding conditions that are potentially reversible may cause delayed decline in allograft function (table 4). When such entities are excluded and a significant decline in FEV1 meets the criteria for BOS, a diagnosis of BOS may be made. However, BOS with obstructive physiology may be distinguished from the recently described entity of restrictive allograft syndrome, which is characterised by restrictive physiology with evidence of allograft parenchymal fibrosis [18]. Therefore, it should be recognised that not all patients in whom a decline in FEV1 and/or airflow obstruction develops necessarily have BOS. Additionally, occult OB may be present in allografts that do not display a significant pattern of FEV1 decline meeting currently accepted criteria for the diagnosis of BOS [19, 20].

The term chronic lung allograft dysfunction (CLAD) has been used in reference to BOS and chronic rejection, and these three terms have been used interchangeably in a number of published manuscripts. However, CLAD is a term that needs to have a rigorous and widely accepted definition. The indiscriminate, interchangeable use of these terms may be perceived as indicating that a decline in FEV1 always indicates the presence of $\mathrm{OB}$ due to chronic rejection, but FEV1 decline may occur for a variety of reasons as stated above.

\section{BOS phenotypes}

The identification of patient groups with specific attributes or patterns of disease may allow the recognition of specific risk factors, pathogenetic disease mechanisms, and/or strategies for treatment and prevention that pertain to an identifiable subset (phenotype) of patients with BOS. Patients with a pattern of early decline in FEV1 that meets BOS criteria may represent a BOS phenotype that has more severe and aggressive $\mathrm{OB}$, which is characterised by rapid progression and poor prognosis [19, 21-23]. However, some patients with rapidly declining lung function may stabilise despite an initial rapid onset and loss of lung function [24]. Another potential BOS phenotype suggested in recent literature consists of recipients with significant bronchoalveolar lavage (BAL) neutrophilia who respond to azithromycin therapy [25, 26]; FEV1 may improve such that the recipient no longer meets the spirometric criteria for BOS. These patients appear to have a reversible, BOS-like syndrome associated with BAL neutrophilia, and the recently published,

\section{TABLE 6 Quality of evidence and strength of recommendations}

Quality of evidence

High

Moderate

Low

Very low

Strength of

recommendations
Evidence includes well-designed, well-conducted randomised trials or meta-analyses of randomised trials, without risk of bias, indirectness, imprecision, inconsistency or publication bias. Alternatively, the evidence may include well-designed, well-conducted observational studies with either a very large effect or at least two of the following: a large effect, dose-response gradient, and/or reverse confounding.

Evidence includes randomised trials or meta-analyses of randomised trials downgraded because of a serious risk of bias, indirectness, imprecision, inconsistency or publication bias. Alternatively, the evidence may include well-designed, well-conducted observational studies upgraded because of a large effect, dose-response gradient or reverse confounding.

Evidence includes well-designed, well-conducted observational studies, or randomised trials or meta-analyses of randomised trials downgraded two levels because of very serious risk of bias, indirectness, imprecision, inconsistency or publication bias.

Evidence consists of case reports, case series or unsystematic clinical observations (i.e. clinical experience or expert opinion).

Strong The committee feels certain that the benefits of the intervention substantially outweigh its risks, burdens and costs.

Weak The committee believes, but is uncertain, that the benefits of the intervention substantially outweigh its risks, burdens and costs. 
randomised prospective clinical trial conducted by VOS et al. [27] suggested that prophylactic administration of azithromycin initiated shortly after transplantation can suppress the development of this syndrome. Patients who meet BOS criteria but do not respond to azithromycin may represent a phenotype with fibroproliferative OB [25]. Nonetheless, distinct phenotypes of BOS that are based upon specific risk factors (table 7) or other parameters have yet to be definitively established.

\section{Risk factors associated with the development of BOS}

Non-minimal (Grade $\geqslant$ A2) acute cellular rejection and lymphocytic bronchiolitis

Grade A2 or above acute cellular rejection on lung biopsy [28] has been linked to subsequent development of BOS [6, 29-36]. Late acute cellular rejection [30, 31, 33] and both increasing frequency and severity of acute cellular rejection $[6,30,33]$ have been found to be risk factors for BOS. Most investigations have found that acute cellular rejection is a major risk factor for BOS even after other clinical events are accounted for by time-dependent Cox regression models and multivariate analyses. Grade B rejection (lymphocytic bronchiolitis (LB)) has also been identified as a risk factor for the development of BOS $[30,33,34,37,38]$.

Our literature search identified no studies that compared augmented immunosuppression with no augmented immunosuppression in patients with non-minimal (Grade $\geqslant$ A2) acute cellular rejection or LB. Such studies will probably never be carried out, because augmented immunosuppression for non-minimal acute cellular rejection or LB is so widely accepted that it is unlikely that a control group is possible (i.e. patients are unlikely to accept the chance of being placed into the no augmented immunosuppression group). However, we identified two relevant studies that support the notion that augmented immunosuppression may decrease the risk of BOS among patients with non-minimal acute cellular rejection or $\operatorname{LB}[33,39]$.

The first study was a case-control study that found patients who developed BOS were more likely to have had inadequate maintenance immunosuppression (cyclosporine, azathioprine, and prednisone, with cyclosporine levels $\left.<200 \mathrm{ng} \cdot \mathrm{mL}^{-1}\right)$ than patients without BOS $(\mathrm{p}<0.0001)$ [33]. The second study was a case series that revealed that augmented immunosuppression was associated with improved or eliminated cellular rejection in $54 \%$ of patients with Grade A2 acute cellular rejection, $48 \%$ with Grade A3 acute cellular rejection, $83 \%$ with Grade A4 acute cellular rejection, and 43\% of patients with LB; patients whose acute cellular rejection or LB neither improved nor resolved usually remained stable [9]. Acute cellular rejection and LB are markers (i.e. surrogate measures) of risk for BOS. The series did not measure how many patients developed BOS, nor did it specify which regimen(s) were used to augment immunosuppression (online supplementary table S2a).

When deciding whether or not augmented immunosuppression is warranted, the likelihood of preventing BOS described above must be balanced against the harms of the increased immunosuppression. This balance will vary depending upon the regimen chosen; however, a short course of systemic steroids is the most common regimen selected [40]. The best evidence regarding the potential adverse effects of a short course of systemic steroids is indirect, extrapolated from randomised trials conducted in patients having an exacerbation of chronic obstructive pulmonary disease (COPD). Such trials have found that short courses of systemic steroids increase the incidence of adverse effects, particularly hyperglycaemia and weight gain (online supplementary table S2a).

Our confidence in the accuracy of the reported effects of augmented immunosuppression on the development of BOS in patients with non-minimal acute cellular rejection or LB (i.e. the quality of

TABLE 7 Risk factors associated with bronchiolitis obliterans syndrome

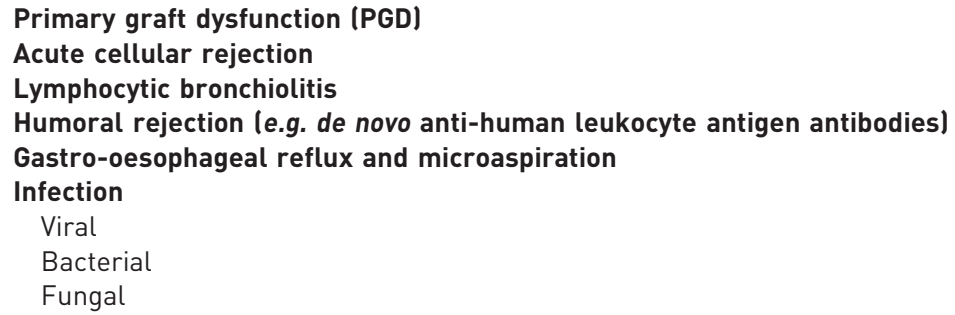

Persistent neutrophil influx and sequestration (bronchoalveolar lavage neutrophilia)

Autoimmunity (collagen V sensitisation) 
evidence) is very low. This is because the estimates are derived from an observational study and a case series, which were limited by risk for bias, small sample sizes, indirectness of the population (included all posttransplant patients rather than specifically patients with non-minimal acute cellular rejection or LB [33]), and indirectness of the outcome (measured the change in acute cellular rejection or LB, rather than the development of BOS [39]). Our confidence in the accuracy of the reported adverse effects of systemic steroids is moderate-to-high because the estimates derive from randomised trials, some of which were limited by a small sample size (online supplementary table S2b).

The committee suggests augmented immunosuppression for patients with non-minimal acute cellular rejection or LB in order to prevent BOS. This is based upon our assessment that the potential benefits of the most common regimen used for augmented immunosuppression (a short course of systemic steroids) outweigh the risks, both in terms of importance (i.e. preventing a life threatening complication versus hyperglycaemia and weight gain) and duration (i.e. the potential benefits are long-term, whereas the risks are only short-term and reversible upon discontinuation of therapy). Moreover, a short course of systemic steroids is not overly costly or burdensome and the committee's collective clinical observations suggest that there is a beneficial effect from such therapy in this population of patients.

The recommendation is conditional because the very low quality of evidence provides little certainty that the desirable consequences of augmented immunosuppression exceed the undesirable consequences. Although the initial approach to augmenting immunosuppression in this setting generally employed by transplant centres worldwide is to give high-dose corticosteroids intravenously (e.g. methylprednisolone at $1000 \mathrm{mg}$ daily for 3 days), other therapies may be required (e.g. lymphodepletion-inducing agents) for a rejection episode. Various changes in the maintenance immunosuppression drug regimen may also be appropriate (see Recommendation 4).

\section{Recommendation 1}

For lung transplant recipients who have non-minimal acute cellular rejection (Grade $\geqslant$ A2) or lymphocytic bronchiolitis on transbronchial lung biopsy specimens, we suggest augmented immunosuppression with a course of systemic steroids to prevent the development of BOS (conditional recommendation, very low quality evidence).

\section{Values and preferences}

This recommendation places a high value on preventing a life-threatening complication of lung transplantation and a lower value on avoiding short-term adverse effects.

\section{Remarks}

A typical course of systemic corticosteroids used to augment immunosuppression in adult recipients is intravenous methylprednisolone $1000 \mathrm{mg}$ daily for 3 days (many centres use $10-15 \mathrm{mg} \cdot \mathrm{kg}^{-1}$ per day for smaller patients).

\section{Minimal (Grade A1) acute cellular rejection}

The significance of minimal acute cellular rejection (Grade A1) is controversial. HopkINs et al. [41] have reported that grade A1 acute cellular rejection remains relatively prevalent on surveillance transbronchial biopsies up to 2 years post-transplant. Available studies suggest that patients with multiple episodes of Grade A1 rejection have an earlier onset of BOS, and a single episode of A1 rejection was found to be independently associated with progression to BOS [42].

Our search identified two observational studies that suggest augmented immunosuppression for minimal (Grade A1) acute cellular rejection may decrease the risk of developing BOS [41, 43]. In one study, intravenous steroids followed by a tapering course of oral steroids was not associated with development of BOS $(p=0.48)$, whereas lack of treatment with systemic steroids was associated with development of BOS $(p=0.01)$ [43]. In the other study, a course of oral steroids reduced progression to higher grades of acute cellular rejection and LB (markers of risk for BOS) by $16.7 \%$ and $15.6 \%$, respectively [41]. Neither of the studies reported adverse effects from the systemic steroids (online supplementary table S3a).

When deciding whether or not augmented immunosuppression is warranted, the likelihood of preventing BOS described above must be balanced against the harms of the increased immunosuppression. The best evidence regarding the adverse effects of short courses of systemic steroids is indirect, extrapolated from randomised trials conducted in patients having an exacerbation of COPD. Such trials have found that short courses of systemic steroids increase the frequency of adverse events, particularly hyperglycaemia and weight gain (online supplementary table S3b). 
Our confidence in the accuracy of the reported effects of augmented immunosuppression on the development of BOS in patients with minimal acute cellular rejection (i.e. the quality of evidence) is very low because the estimates derive from observational studies that are limited by a risk for bias, indirectness of either the outcome (measured progression to higher grades of acute cellular rejection and LB rather than development of BOS [41]) or use of an indirect comparator (looked at associations in treated and untreated patients separately, rather than directly comparing treatment with no treatment [43]). Our confidence in the accuracy of the reported adverse effects of systemic steroids is moderate-to-high due because the estimates derive from randomised trials, some of which were limited by a small sample size (online supplementary table S3b).

The committee suggests that patients with clinically significant minimal acute cellular rejection be treated with a course of systemic steroids. This reflects the committee's judgment that the possible benefits (i.e. preventing BOS) of therapy exceed the risks (i.e. hyperglycaemia and weight gain), cost and burden in such patients. The rationale for treating minimal acute cellular rejection with systemic steroids is similar to that provided earlier for treating non-minimal acute cellular rejection and LB with systemic steroids. The recommendation is conditional because the balance of desirable versus undesirable consequences is uncertain due to the very low quality of the evidence, and the uncertainty is reinforced by the committee's clinical experience. By contrast, for clinically stable patients with grade A1 acute cellular rejection on a surveillance biopsy, the decision about whether to immediately treat the patient with augmented immunosuppression or observe and repeat the biopsy before reaching the decision to augment immunosuppression should be made on a case-by-case basis.

Recommendation 2

For lung transplant recipients who have clinically significant minimal acute cellular rejection (Grade A1) on transbronchial lung biopsy specimens, we suggest augmented immunosuppression with a course of systemic steroids to prevent the development of BOS (conditional recommendation, very low quality evidence).

Values and preferences

This recommendation places a high value on preventing a life-threatening complication of lung transplantation and a lower value on avoiding short-term side-effects.

\section{Remarks}

We consider Grade A1 acute cellular rejection to be clinically significant if it is associated with clinical findings, such as symptoms (e.g. dyspnoea, fatigue or new-onset cough) or objective measurements (e.g. decline in FEV1 or oxyhaemoglobin desaturation with ambulation), that suggest the presence of allograft dysfunction. A typical course of systemic steroids used to augment immunosuppression in adult recipients is intravenous methylprednisolone $1000 \mathrm{mg}$ daily for 3 days (many centres use $10-15 \mathrm{mg} \cdot \mathrm{kg}^{-1} \mathrm{per}$ day for smaller patients).

\section{Other risk factors}

Other risks factors for the development of BOS are discussed in the online supplementary material and include the presence of anti-HLA antibodies [44-48], PGD [49-52] and the presence of significant (abnormal in degree) GOR [53-55]. Additional associated risk factors include viral infection [29, 31, 32, 56-64], bacterial infection [65-68] and fungal infection [69-71]. Cytomegalovirus infection engages both the innate and adaptive components of immunity and causes upregulation of HLA class I and class II antigens on epithelial cells $[72,73]$, and stimulates and augments the generation of allogeneic immune responses and proinflammatory cytokines [72, 74]. Transient bacterial airway colonisation can significantly increase BAL neutrophils and other indicators of lung inflammation [75]. Bотна et al. [53] examined 155 consecutive lung transplants and reported that de novo allograft colonisation with Pseudomonas aeruginosa was strongly associated with developing BOS within 2 years of transplant. Vos et al. [54] reported that persistent Pseudomonas colonisation was an even greater risk for BOS than de novo colonisation. Additionally, GOTTLIEB et al. [55] found that persistent allograft colonisation with Pseudomonas in recipients with cystic fibrosis significantly increased the prevalence of BOS. VALENTINE et al. [69] identified fungal pneumonia or pneumonitis as an independent predictor of subsequent BOS, and WEIGT et al. [70] reported that Aspergillus colonisation was independently associated with the subsequent development of BOS.

A prospective study that monitored peripheral blood mononuclear cell responses in 54 lung transplant recipients over a 7-year period showed a strong association of collagen V-specific responses with the incidence (hazard ratio (HR) 5.4 for BOS Grade 1 and HR 9.8 for BOS Grade 2) and severity of BOS [13], and induction of collagen $\mathrm{V}$ reactivity has been associated with abnormal GOR and the development of BOS [76]. In addition, SAINI et al. [77] found a strong association between the appearance of donor-specific 
anti-HLA antibodies and the detection of antibodies directed against self-antigens (collagen V and K- $\alpha 1$ tubulin) in a retrospective analysis of 42 lung transplant recipients with BOS.

Numerous studies have shown evidence of neutrophil recruitment and activation when BAL was performed in recipients with acute rejection, infection and/or BOS [78-81]. NEUROHR et al. [82] found that BAL neutrophilia was predictive of subsequent BOS. SCHLOMA et al. [83] also reported that increased BAL neutrophils were associated with early onset BOS, and subsequent investigations by GOTTLIEB et al. [26] and Vos et al. [25] have also linked BAL neutrophilia to BOS.

Recommendations for mitigating risk factors for BOS are beyond the scope of this guideline, but may be addressed in future guidelines.

\section{Diagnosis of BOS}

An FEV1 decline should trigger concern that graft dysfunction and possibly BOS is evolving, and considerable allograft damage from evolving BOS may have already occurred by the time FEV1 has declined by $20 \%$ from its baseline value. When clinically stable patients develop symptoms (e.g. dyspnoea, cough, fatigue or fever) and/or signs (decline in FEV1 on home spirometry or at clinic visit follow-up evaluation) that may indicate allograft dysfunction, a comprehensive evaluation to determine the cause is typically initiated (fig. 2). This usually includes a routine evaluation in the clinic, followed by specific testing (imaging, confirmatory spirometry and bronchoscopy, as indicated) to identify a specific cause or causes of lung function decline. If BOS appears to be the cause of lung function decline, the treatment approaches discussed in the following section can be considered.

LAMA et al. [84] found that the probability of testing positive for BOS Grade 0-p by the FEV1 criterion was $71 \%$ at 2 years before the onset of BOS and the specificity of the FEV1 criterion was $93 \%$ in single lung transplant recipients. HACHEM et al. [85] reported a positive predictive value of $79 \%$ and negative predictive value of $82 \%$ for stage 0 -p by FEV1 criteria in 203 adult bilateral lung transplant recipients, but the FEF $25-75 \%$ 0 - $p$ criterion had poor predictive value. The prevalence of BOS in the study was $41-63 \%$ depending on the criteria used to define BOS. By contrast, NATHAN et al. [86] found $80 \%$ sensitivity and $82.6 \%$ specificity of the FEF25-75\% 0-p criterion in a cohort of 43 single lung transplant recipients. Differences between these studies may be related to different statistical techniques, sample size and follow-up time.

High-resolution computed tomography (HRCT) may detect diagnostically useful pleuroparenchymal changes and/or air trapping to which routine chest radiography is insensitive [87-94]. Bronchoscopy with transbronchial lung biopsy (TBLB) and BAL is useful to detect infection or other entities that may be the cause of functional decline. Although changes consistent with OB may be obtained via TBLB, nonsurgical lung biopsy is insensitive, and a lack of changes of OB on TBLB has poor predictive value. Although many potential biomarkers of BOS have been reported in the literature, none have been validated as having adequate sensitivity and specificity.

Evaluation and/or screening of children for changes in lung function is particularly challenging, and children under 4 years of age may be unable to perform spirometry, necessitating specialised approaches $[95,96]$. Paediatric centres are likely to use alternative lung function measurements or imaging modalities such as ventilation/perfusion scanning and inspiratory/expiratory HRCT scanning to enhance the ability to detect the presence of airflow obstruction. Because TBLB is difficult to perform in infants and small children, many paediatric centres use surgical lung biopsy to confirm a diagnosis of suspected OB [97].

Diagnostic recommendations for BOS are beyond the scope of this guideline, but may be addressed in future guidelines.

\section{Treatment and prevention of BOS}

Intensified pharmacological immunosuppression has little effect on established BOS in the absence of confounders such as acute cellular rejection, antibody-mediated rejection or lack of BAL neutrophilia.

\section{Long-term high-dose corticosteroids}

Sustained treatment with high-dose corticosteroids $\left(\geqslant 30 \mathrm{mg} \cdot\right.$ day $^{-1}$ prednisone or an equivalent) has not been shown to improve BOS, and such therapy is associated with numerous and frequently severe sideeffects [98]. Our search identified a case series of 10 patients with lung function decline consistent with BOS [99]. All 10 patients exhibited progressive lung function decline despite receiving high-dose methylprednisolone. Adverse effects of the sustained high-dose methylprednisolone were not reported in the case series; however, there is indirect evidence from patients with chronic lung diseases that sustained high-dose corticosteroids are harmful to patients. Specifically, sustained high-dose corticosteroids increase the incidence of osteoporotic fractures, cataracts and dyspepsia. The finding that sustained high-dose 


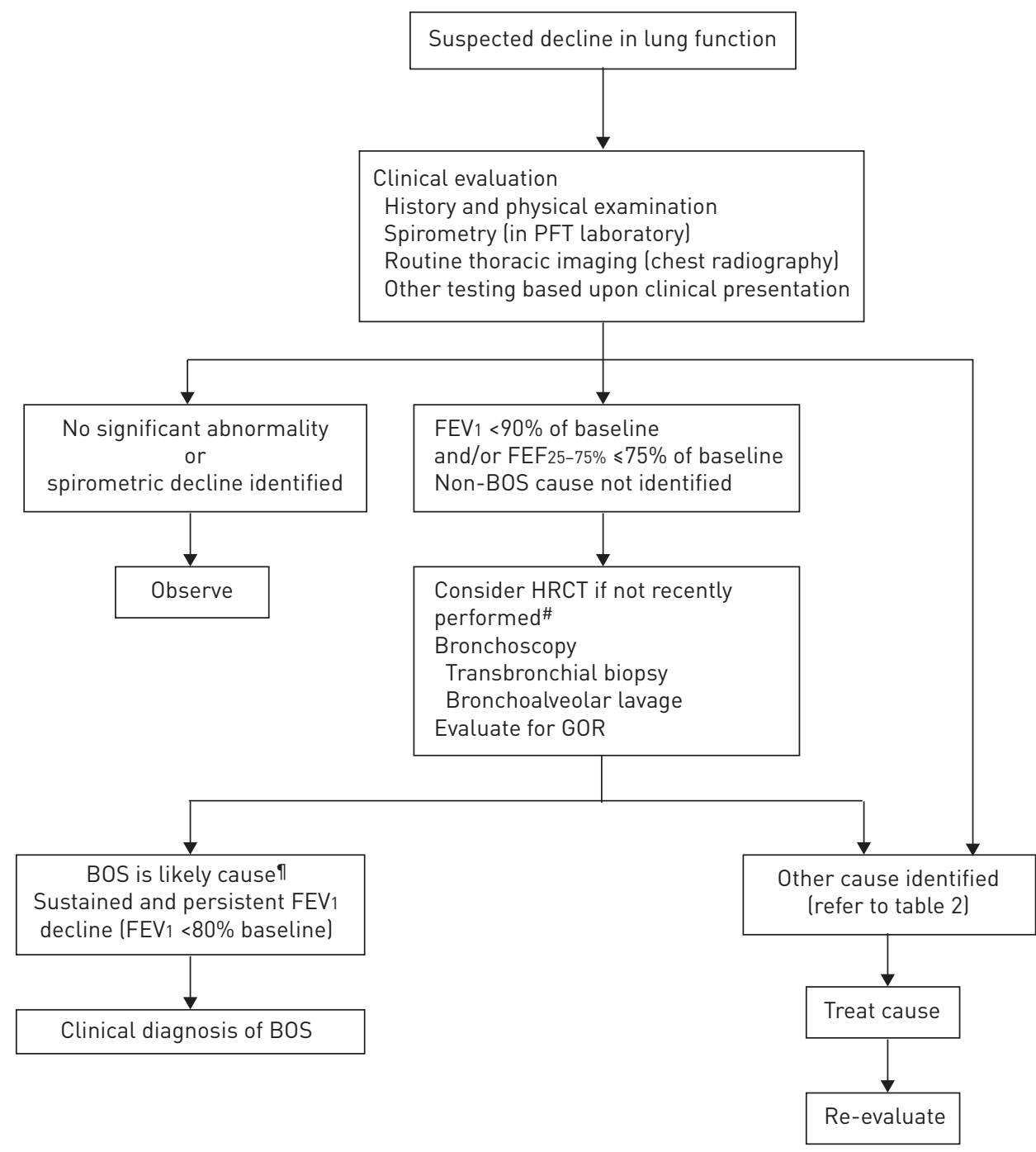

FIGURE 2 Algorithm for clinical evaluation of suspected bronchiolitis obliterans syndrome (BOS). This algorithm is a description of the collective clinical practices of the committee members. It is not based upon systematically developed evidence-based diagnostic recommendations. PFT: pulmonary function test; FEV1: forced expiratory volume in $1 \mathrm{~s}$; FEF25-75\%: forced expiratory flow at 25-75\% of forced vital capacity; HRCT: high-resolution computed tomography; GOR: gastro-oesophageal reflux. ${ }^{\#}$ : obtain both inspiratory and expiratory views to evaluate for air trapping; ${ }^{\text {i }}$ : the presence of bronchiolitis obliterans lesions on lung biopsy (if obtained) is considered diagnostic and HRCT findings consistent with bronchiolitis (e.g. air-trapping) are supportive, restrictive allograft syndrome is an alternative diagnostic consideration if a restrictive pattern is found on pulmonary function testing.

corticosteroids induce no beneficial effects on lung function, but cause numerous serious adverse effects, is supported by the collective clinical observations of the committee members (online supplementary table S4a).

Our confidence in the accuracy of the reported effects of sustained high-dose corticosteroids on the lung function of patients with a decline in FEV1 consistent with BOS (i.e. the quality of evidence) is very low, because the estimates derive from one small case series. Our confidence in the accuracy of the reported adverse effects of systemic steroids varies from low to high depending upon the outcome, because the estimates derive from observational studies with one outcome (osteoporotic fractures) upgraded as there was a dose-response gradient and an effect was seen even though confounders would tend to underestimate the effect (online supplementary table S4b).

We suggest not using sustained high-dose systemic corticosteroids for patients who have a decline in FEV1 consistent with BOS given the lack of proven benefit and the potential for serious adverse effects. The recommendation is conditional because the evidence provides very low confidence in the effect of sustained high-dose systemic corticosteroids. 


\section{Recommendation 3}

For lung transplant recipients who develop a decline in FEV1 consistent with the onset of BOS, we suggest that clinicians do not use long-term, high-dose corticosteroids (conditional recommendation, very low quality evidence).

Values and preferences

This recommendation places a high value on avoiding harmful effects due to ineffective therapies.

Remarks

We define sustained administration of high-dose corticosteroid as $\geqslant 30 \mathrm{mg} \cdot \mathrm{day}^{-1}$ of prednisone or an equivalent formulation.

\section{Converting cyclosporine to tacrolimus}

If patients are receiving cyclosporine A-based immunosuppression, switching from cyclosporine A to tacrolimus has been reported to slow lung function loss in a number of case series [99-108]. However, no randomised trials have been performed to support this switch.

Our search identified 10 case series that described the effects of converting cyclosporine to tacrolimus in lung transplant patients with BOS. Six of the series reported mitigation of lung function decline following conversion, while the remaining four series reported improvement of lung function following conversion. Most of the case series did not mention adverse effects; however, those that evaluated nephrotoxicity or hyperglycaemia reported a frequent rise in the serum creatinine and glucose levels. None of the case series described infections or malignancy (online supplementary table S5a).

There is indirect evidence from randomised trials of patients who have undergone renal transplantation that indicates tacrolimus does not increase the risk of infection, malignancy, nephrotoxicity or hyperglycaemia when compared with cyclosporine [109]. We have no reason to believe that the adverse effects of tacrolimus and cyclosporine are different in lung transplant patients compared with renal transplant patients.

Our confidence in the accuracy of the reported effects of converting cyclosporine to tacrolimus on lung function, nephrotoxicity and hyperglycaemia (i.e. the quality of evidence) is very low because the estimates derive from small case series (online supplementary table S5b).

In lung transplant recipients who develop BOS while receiving a maintenance immunosuppression regimen that includes cyclosporine, we suggest that the cyclosporine is converted to tacrolimus. This reflects the committee's opinion that the likely benefits of mitigation or reversal of lung function decline outweighs the risks of an increase in the serum creatinine and/or glucose levels. The recommendation is conditional because the balance of desirable and undesirable consequences is very uncertain due to the very low quality of the available evidence.

\section{Recommendation 4}

For lung transplant recipients who develop BOS while receiving chronic immunosuppression with a regimen that includes cyclosporine, we suggest switching the cyclosporine to tacrolimus (conditional recommendation, very low quality evidence).

Values and preferences

This recommendation places a higher value on mitigation of lung function decline and a lower value on avoiding nephrotoxicity and hyperglycaemia.

\section{Remarks}

The conversion of cyclosporine to tacrolimus is generally performed by stopping cyclosporine and initiating tacrolimus while transiently increasing maintenance corticosteroid dosing until tacrolimus blood levels are ascertained to have reached the desired target range. The target for therapeutic trough blood levels of tacrolimus is generally considered to range from 5 to $15 \mathrm{ng} \cdot \mathrm{mL}^{-1}$ for patients who are $\geqslant 18$ years of age once a steady state has been attained.

\section{Azithromycin}

Beneficial effects have been reported for approximately $35-40 \%$ of lung transplant recipients treated with azithromycin [25, 26, 110-116]. Complete reversal of FEV1 decline may occur in some patients, and patients with BAL neutrophilia appear to represent a subset of patients that are particularly likely to respond to azithromycin therapy $[25,26,114]$. 
Our literature search identified 10 studies (one observational study and nine case series) that described the effects of azithromycin on the lung function of lung transplant patients with BOS. The studies found that $30-83 \%$ of patients had improved lung function (defined as an increase in FEV1 of $\geqslant 10 \%$ ) after receiving azithromycin, even though the mean FEV1 did not increase in some studies because nonresponders continued to have lung function decline. In addition, two observational studies were identified that described the effects of azithromycin on mortality in lung transplant patients with BOS. Both studies found that early treatment was associated with decreased mortality in some patients. In one study, lung transplant patients with BOS Stage 1 who received azithromycin had lower mortality than those who did not receive azithromycin (HR $0.29,95 \%$ CI $0.11-0.82$ ). The mortality decrease was not seen among patients with BOS Stage 2 [116]. In the other study, $40 \%$ of patients responded to azithromycin and those patients had a reduction in mortality (HR 0.96, 95\% CI 0.95-0.98). Responders tended to receive azithromycin earlier post-transplantation [25]. Most of the studies did not mention adverse effects; however, the most common adverse effects reported were nausea, diarrhoea, dyspepsia and colitis, occurring in fewer than $5 \%$ of patients (online supplementary table S6a).

There is indirect evidence from randomised trials in other conditions that probably better estimates the incidence of adverse effects from azithromycin. A meta-analysis of 12 randomised trials with 1406 patients who received azithromycin to treat an acute lower respiratory tract infection found that 244 (17.9\%) out of 1363 patients developed an adverse event [117]. Most of the adverse events were minor nausea and diarrhoea. Neither the studies identified by our systematic review, nor the meta-analysis of azithromycin for lower respiratory infections, reported fatal cardiac arrhythmias. However, there is other evidence that azithromycin is associated with fatal cardiac arrhythmias. In an observational study that looked at more than one million instances of taking azithromycin, patients who took azithromycin were more likely to suffer a fatal cardiac arrhythmia than those who did not take an antibiotic (risk ratio $2.85,95 \%$ CI 1.13-7.24) [118]. The absolute risk of a fatal cardiac arrhythmia during azithromycin therapy was small (1.1 cases per 1000 person-years) and the risk was not increased compared with patients who took an alternative antibiotic (risk ratio 0.93, 95\% CI 0.56-1.55) [118]. These findings were supported by another study conducted by the maker of azithromycin [119]. There is also evidence from a randomised trial that patients with COPD who are treated with chronic azithromycin therapy are more likely to experience a decrement in hearing and colonisation with azithromycin-resistant organisms [120].

Our confidence in the accuracy of the reported effects of a trial of azithromycin on lung function, survival, gastrointestinal distress and allergic reactions is very low, because the estimates derive primarily from case series and a few small observational studies (online supplementary table S6b). Similarly, our confidence in the accuracy of the reported effects of a trial of azithromycin on fatal cardiac arrhythmias is very low because it derives from observational studies in a different patient population. The relevance of the reported fatal cardiac arrhythmias to lung transplant patients is uncertain, because lung transplant recipients are uniformly screened to rule out the presence of significant coronary disease or cardiac dysfunction prior to being listed for transplantation. Finally, our confidence in the accuracy of the reported effects of a trial of azithromycin on hearing loss and the acquisition of colonisation with azithromycin-resistant organisms is moderate, because it derives from a single randomised trial with a different patient population.

We suggest a trial of azithromycin in lung transplant recipients who develop BOS. This reflects the committee's judgment that the importance of improved lung function and decreased mortality exceed the risk of minor gastrointestinal distress, decreased hearing, colonisation with azithromycin-resistant organisms, rare fatal cardiac arrhythmias and rare allergic reactions. The recommendation is conditional because our very low-to-moderate confidence in the reported effects provides limited certainty that the benefits (improved lung function and decreased mortality) exceed the potential adverse events (nausea, diarrhoea, fatal cardiac arrhythmias, decreased hearing, colonisation with azithromycin-resistant organisms and allergic reactions).

\section{Recommendation 5}

For lung transplant recipients who develop a decline in FEV1 consistent with the onset of BOS, we suggest a trial of azithromycin (conditional recommendation, very low quality evidence).

Values and preferences

This recommendation places a high value on preventing lung function deterioration and possibly reducing mortality, and a lower value on avoiding adverse effects.

Remarks

Azithromycin is generally administered orally at $250 \mathrm{mg}$ per day for 5 days and then $250 \mathrm{mg}$ three times per week. We define a trial of azithromycin as treating continuously with azithromycin for a minimum of 
3 months. Additionally, it is unclear 1) whether azithromycin should be continued long-term if a beneficial response is observed, or 2) whether it should be discontinued if lung function does not show improvement during follow-up clinical evaluation.

\section{Anti-reflux surgery}

Abnormal GOR (identified by oesophageal $\mathrm{pH}$ probe in the majority of studies) is highly prevalent in patients with advanced lung disease and in lung transplant recipients [65, 66, 68, 121-126], and it has been implicated as a risk factor for BOS $[65,66,68,127,128]$. For this reason, committee members routinely test patients with new onset BOS for GOR. Proximal gastrointestinal tract motility studies and $\mathrm{pH} / \mathrm{impedance}$ testing can be used to diagnose motility abnormalities and abnormal acid and/or non-acid GOR [129]. In addition, examination of BAL for markers of aspiration (e.g. oil red $\mathrm{O}$ staining and determination of a lipid index, BAL fluid pepsin and BAL fluid bile acids) has been reported as useful for the detection of microaspiration of refluxed gastro-oesophageal material [66, 130, 131]. However, additional studies correlating BAL markers of aspiration with GOR and BOS are needed to facilitate selection of recipients with BOS who may benefit from interventions such as laparoscopic fundoplication.

Anti-reflux surgery (e.g. Nissen fundoplication and Toupet fundoplication) can be performed safely on lung transplant candidates with advanced lung disease or lung transplant recipients with documented abnormal GOR [123, 124, 127-129, 132-138], thereby preventing reflux, aspiration of gastric secretions and related sequelae.

Our literature search identified three observational studies and five case series that reported the effects of anti-reflux surgery on lung function and mortality in lung transplant recipients. Seven out of eight studies found that the FEV1 improved following anti-reflux surgery (including the two studies that looked specifically at lung transplant recipients with BOS) and the two studies that described long-term survival both reported improved survival after anti-reflux surgery (online supplementary table S7a).

With respect to the safety of anti-reflux surgery, one observational study and three case series reported a complication rate of less than $5 \%$ when pooled. Similarly, one observational study and six case series reported a perioperative mortality rate of less than $1 \%$ when pooled. Three case series reported that $6-14 \%$ of patients develop post-operative dysphagia (online supplementary table S7a). There is also indirect evidence about the safety of anti-reflux surgery that can be extrapolated from nontransplant patients with GOR. Consider the following examples. In one systematic review, a meta-analysis of three randomised trials with 111 nontransplant patients undergoing Nissen fundoplication found that intra-operative complications (e.g. bleeding, liver and spleen capsule tears, and stomach perforation) occurred in $18 \%$ of patients and dysphagia developed in 14\% [139]. There were no perioperative deaths. In a similar systematic review, a meta-analysis of five randomised trials with 388 nontransplant patients with GOR found that perioperative morbidity occurred in $14 \%$ of patients and dysphagia developed in $17 \%$ of patients [140]. There were no perioperative deaths.

There are conflicting data regarding whether lung transplant patients undergoing anti-reflux surgery have a higher incidence of nonfatal perioperative complications than nontransplant patients undergoing anti-reflux surgery. One retrospective cohort study of 52 patients found no differences in estimated blood loss, duration of surgery, length of hospital stay, complications or readmission rate [135]. By contrast, another retrospective cohort study of 28 patients found a longer post-operative hospital stay ( 2.9 versus 0.7 days) and higher 30-day readmission rate (25\% versus 3.2\%) among lung transplant patients than nontransplant patients [23].

Our confidence in the accuracy of the reported effects of anti-reflux surgery on lung function, mortality and peri-operative complications is very low, because they derive from observational studies and cases series limited by indirectness of the population (most studies looked at lung transplant patients with GOR in general, rather than lung transplant patients with GOR and probable BOS) and imprecision of the reported effects owing to few observed events (online supplementary table S7b).

We suggest that lung transplant patients with GOR who develop a decline in FEV1 consistent with the onset of BOS be referred for potential fundoplication of the gastro-oesophageal junction. This is based upon the committee's observation that fundoplication may improve lung function and decrease mortality with low risk for perioperative complications. Our recommendation is conditional because the very low quality of the available evidence provides little certainty that the desirable consequences outweigh the undesirable ones.

\section{Recommendation 6}

For lung transplant recipients who develop a decline in FEV1 consistent with the onset of BOS and have confirmed GOR, we suggest referral to an experienced surgeon to be evaluated for potential fundoplication of the gastro-oesophageal junction (conditional recommendation, very low quality evidence). 
Values and preferences

This recommendation places a high value on reducing the risk of lung function deterioration and possibly mortality, and a lower value on avoiding surgical complications.

Remarks

Nissen fundoplication has been more extensively studied than Toupet fundoplication; however, we have no reason to believe that one is superior to the other and feel that the choice of the surgical technique should remain at the surgeon's discretion.

\section{Re-transplantation}

A number of single-centre observational studies and case series have evaluated the outcomes of retransplantation [141-147]. Survival rates have improved significantly in the modern era [145, 146], and outcomes following re-transplantation for carefully selected patients with BOS (ambulatory patients selected via the same process used for first-time transplantation) may approach those of first-time lung transplants if performed by experienced centres [142, 148].

Our literature search identified four observational studies and three case series that reported the effects of re-transplantation on lung function and survival [141-147]. With respect to lung function, freedom from BOS following re-transplantation surgery for BOS was reported to be $85-90 \%$ at 1 year, $70-77 \%$ at 2-3 years and $50-77 \%$ at $4-5$ years. Patients who underwent re-transplantation due to BOS had a higher risk of recurrent BOS than patients who underwent re-transplantation for other reasons, but it is uncertain whether they also had a higher risk for BOS than patients who underwent first-time lung transplantation because the results were conflicting. Survival following re-transplantation for BOS was reported to be $60-78 \%$ at 1 year, $53-64 \%$ at 2 years and $44-61 \%$ at 5 years. Survival was higher among patients who underwent re-transplantation for BOS than among patients who underwent re-transplantation for other reasons, but it was lower than that of patients undergoing primary lung transplantation (online supplementary table S8a).

The safety of re-transplantation has not been well studied. We identified no studies that reported perioperative morbidity and only one observational study that reported perioperative mortality. In that study [147], there were 39 (10\%) deaths within 180 days among the 389 patients who underwent retransplantation. The causes of death were infection, respiratory failure and multi-organ system failure. Patients undergoing re-transplantation had an increased risk for death after the procedure compared with patients who underwent primary transplantation (online supplementary table S8a). Our confidence in the reports of freedom from BOS, survival and perioperative mortality (i.e. quality of evidence) is very low because the estimates derive from observational studies and case studies with limitations, usually indirectness of the population (online supplementary table S8b).

We suggest referring patients who develop refractory end-stage BOS to a transplant surgeon to be evaluated for re-transplantation for two reasons. First, re-transplantation is usually the only hope for survival since such patients have already failed alternative interventions. Second, re-transplantation probably improves survival. Survival without re-transplantation has been reported to be $51 \%$ at 3 years for all BOS stages [32] and is certainly much lower among those whose BOS is severe enough to require re-transplantation (patients are usually classified as Stage 3 when referred for re-transplantation), as suggested by the observed two- to three-fold increased risk of death with progression of each grade of BOS to a higher grade (e.g. stage 1 to 2 and then stage 2 to 3) [23,32]. It is noteworthy that survival has recently been reported to differ for early versus late onset BOS with a progressive reduction in mortality risk if BOS onset occurs later (e.g. $<1$ year, 1-2 years, 2-3 years or $>3$ years) after transplantation versus earlier onset [149], suggesting that recipients with early onset BOS are at especially high risk for a poor survival outcome. Our recommendation is conditional because the very low quality of the available evidence provides little certainty that the desirable consequences (i.e. potential mortality reduction) outweigh the undesirable consequences (i.e. increased risk of recurrent BOS, perioperative mortality and resource utilisation); therefore, the appropriate course of action likely depends upon the clinical context.

\section{Recommendation 7}

For lung transplant recipients who have developed end-stage BOS refractory to other therapies, we suggest referral to a transplant surgeon to be evaluated for re-transplantation (conditional recommendation, very low quality evidence).

\section{Values and preferences}

This recommendation places a high value on avoiding surgical complications (e.g. mortality), recurrent BOS and resource utilisation. 
Remarks

The selection process for re-transplantation is the same as that used for first-time lung transplantation.

\section{Key unanswered questions and specific research needs}

Key unanswered questions and research needs are listed in table 8. Despite the identification of numerous risk factors that are associated with the onset of BOS, the specific mechanisms by which BOS is initiated in the lung allograft remain unknown, and key mediators of airway injury that can be targeted by specific therapies need to be identified. In addition, lung function decline characterised as BOS by the FEV1 criterion may be caused by a number of different mechanisms. Additional research is needed to understand and characterise the complex histopathological changes that may be present in dysfunctional lung allografts and to identify and characterise BOS phenotypes that can be distinguished on the basis of clinical, histopathological and/or pathogenic mechanisms. As treatments become available that may have a therapeutic effect on the course of BOS, reliable biomarkers of early disease need to be identified to optimise the impact of therapeutic interventions. Guidelines addressing how to detect abnormal GOR, select patients for anti-reflux surgery and select the appropriate type of anti-reflux surgery to prevent or treat BOS have yet to be established. In addition, optimal approaches to allograft surveillance (e.g. the role of bronchoscopy with transbronchial biopsies in clinically stable lung transplantation recipients, screening for de novo antiHLA antibodies and the presence of humoral rejection) have yet to be determined.

A number of complex issues need to be resolved by additional research. These include how to deal with categorisation and management of recipients whose spirometry values fluctuate considerably over time, classification of patients who meet criteria for the diagnosis of BOS but subsequently experience a significant improvement in response to therapy (e.g. azithromycin or fundoplication) leading to clinical and functional (FEV1) improvement such that the criteria for BOS are no longer met, and the issue of FEV1 decline that meets BOS criteria when evidence of allograft infection is present. Indeed, infection (e.g. chronic bacterial infection) and OB may coexist, and a diagnosis of BOS may only become apparent after a period of time has elapsed (e.g. 3-6 months) when the infection has cleared or been adequately suppressed and allograft function still does not significantly improve. Additionally, the role of inhaled antibiotics

TABLE 8 Key unanswered questions and research needs

\section{Unanswered questions}

1) What are the roles and mechanisms of alloimmune and autoimmune responses in BOS pathogenesis?

2) Does antibody-mediated rejection play a role in BOS onset and progression?

3) What is the significance of the appearance of de novo anti-HLA antibodies in BOS pathogenesis, and when and how should screening and treatment for anti-HLA antibodies be performed?

4) Can specific biomarkers identify and reliably predict increased risk for the development of BOS, and can such biomarkers be used to detect the early (subclinical) onset of BOS?

5) Can specific BOS phenotypes be identified that are useful for predicting prognosis and response to therapy?

6) Which specific agent or combinations of post-transplant immunosuppressive agents are most likely to prevent BOS and improve allograft and patient survival?

7) Does any early, specific therapy significantly alter the natural history of BOS?

8) When lung retransplantation is performed for end-stage BOS, is the retransplanted lung at increased risk for the development of rejection and/or OB?

9) Can patients who are more tolerant to their grafts and, therefore, require less intense immunosuppression be identified?

10) Can induction of tolerance to self-antigens (e.g. collagen V) or strategies to augment regulatory T- or B-cells to promote and maintain tolerance diminish risk for BOS?

11) Will the use of ex vivo lung perfusion (EVLP) techniques to condition the lung allograft diminish the risk of developing BOS?

12) What is the optimal frequency for obtaining spirometry to assist in the early detection of evolving BOS?

\section{Research needs}

1) Multi-centre clinical investigations are needed to identify and assess risk factors for BOS.

2) Multi-centre clinical trials are needed to evaluate potential therapeutic interventions to treat BOS as well as strategies to prevent its onset.

3) Additional studies of mechanisms and phenotypes (animal models and lung allograft recipients) are needed.

4) Guidelines for optimal testing for abnormal GOR and the selection of patients (and procedure) for anti-reflux surgery to prevent or treat BOS.

5) Identification of optimal approaches to allograft surveillance (e.g. the role of bronchoscopy with transbronchial biopsies in clinically stable lung transplant recipients, screening for de novo anti-HLA antibodies and the presence of humoral rejection).

6) Improved animal and other laboratory models of OB to better understand its pathogenesis and identify key mediators of airway inflammation and fibrosis.

BOS: bronchiolitis obliterans syndrome; HLA: human leukocyte antigen; OB: obliterative bronchiolitis; GOR: gastro-oesophageal reflux. 
used to prevent or suppress bacterial infection in the prevention or management of BOS needs to be determined.

Advances in understanding the effects of allograft cellular senescence (accelerated ageing) on allograft function and BOS risk are needed, and a better understanding of the role of interleukin-17 [150-156], autoimmune pathways, regulatory lymphocyte populations [157-163] and neutrophil responses, as well as mechanisms by which the hypothetical phenomenon of epithelial-mesenchymal transition (which remains hypothetical and has not been well validated in humans) [164-167] leads to airway fibrosis, may lead to novel therapies to prevent and treat BOS. Improved animal models of OB are needed and are likely to be useful in improving our understanding of the role of these and other phenomena in the initiation, progression, prevention and treatment of $\mathrm{OB}$ following lung transplantation.

New methods of allograft conditioning, such as ex vivo lung perfusion (EVLP) [168-172] may lead to improved early allograft dysfunction, diminish the risk of PGD, and decrease both the incidence and severity of BOS, but data to determine the impact of EVLP on BOS risk are not yet available. Intensified immunosuppression with total lymphoid irradiation (TLI) $[173,174]$ or extracorporeal photopheresis (ECPP) $[175,176]$ suggest that these interventions may have a significant, beneficial impact on lung function decline due to BOS, but these interventions can have significant adverse effects and may be associated with significant economic issues, and additional clinical research is required to establish the efficacy of these interventions. Single-centre studies have suggested that other immunosuppressive therapies, such as sirolimus [177], alemtuzumab [178, 179] or anti-thymocyte globulin [180], may play a role in the prevention or management of BOS, but additional research is required to evaluate the utility of TLI, ECPP or other changes in chronic maintenance immunosuppression by using alternative immunosuppressive agents (e.g. the mTOR inhibitor, everolimus) to prevent or manage BOS. Finally, multicentre trials are needed to better establish optimal regimens for the induction and maintenance of immunosuppression that can adequately prevent both acute and chronic allograft dysfunction yet not lead to excessive risk for infection or other untoward consequences, and collaboration among lung transplant centres to provide adequately powered clinical trials will greatly facilitate the identification of specific risk factors and interventions to both treat and prevent BOS.

\section{Conclusions}

This guideline is intended to enhance the understanding of the diagnosis and management of BOS by transplant physicians and other clinicians, and to assist them in the making appropriate clinical decisions when evaluating patients in whom a diagnosis of BOS is suspected. The recommendations in this guideline were informed by a combination of published literature and the clinical observations of experts in the field of lung transplantation. Therefore, they can be used worldwide to help standardise the management of BOS. It is hoped that this guideline will provoke and facilitate future clinical studies in lung transplant recipients who develop delayed loss of allograft function.

\section{Acknowledgements \\ The ISHLT/ATS/ERS BOS Task Force Committee members are as follows.}

Chair: Keith C. Meyer (School of Medicine and Public Health, University of Wisconsin-Madison, Madison, WI, USA).

Co-Chairs: Ganesh Raghu (School of Medicine, University of Washington, Seattle, WA, USA); Geert M. Verleden (University of Leuven, Leuven, Belgium); Paul A. Corris (Freeman Hospital, Newcastle upon Tyne, UK); Allan R. Glanville (The Lung Transplant Unit, St Vincents Hospital, Sydney, Australia); Paul Aurora (Great Ormond Street Hospital for Children, London, UK); Jim J. J. Egan (Mater Misericordiae University Hospital, Dublin, Ireland).

Committee members: Selim Arcasoy (Columbia University, New York, NY, USA); Robert M. Aris (University of North Carolina, Chapel Hill, NC, USA); Robin K. Avery (Johns Hopkins University, Baltimore, MD, USA); John Belperio (University of California Los Angeles, Los Angeles, CA, USA); Juergen Behr (Ruhr University Bochum, Bochum, Germany); Sangeeta Bhorade (Northwestern University, Chicago, IL, USA); Annette Boehler (University of Zurich, Zurich, Switzerland); Cecilia Chaparro (University of Toronto, Toronto, ON, Canada); Jason D. Christie (University of Pennsylvania, Philadelphia, PA, USA); Lieven J. Dupont (Katholieke Universiteit Leuven, Leuven, Belgium); Marc Estenne (Hospital Erasme, Brussels, Belgium); Andrew J. Fisher (University of Newcastle, Newcastle, UK); Edward R. Garrity, Jr (University of Chicago, Chicago, IL, USA); Denis Hadjiliadis (University of Pennsylvania, Philadelphia, PA, USA); Marshall I. Hertz (University of Minnesota, Minneapolis, MN, USA); Shahid Husain (University of Toronto, Toronto, ON, Canada); Martin Iversen (Rigshospitalet, Copenhagen, Denmark); Shaf Keshavjee (University of Toronto, Toronto, ON, Canada); Vibha N. Lama (University of Michigan, Ann Arbor, MI, USA); Deborah J. Levine (University of Texas San Antonio, San Antonio, TX, USA); Stephanie M. Levine (University of Texas San Antonio, San Antonio, TX, USA); James C. Lee (University of Pennsylvania, Philadelphia, PA, USA); Gilbert Massard (University of Strasbourg, Strasbourg, France); Atul C. Mehta (Cleveland Clinic, Cleveland, OH, USA); Steven D. Nathan (Inova Fairfax Hospital, Falls Church, VA, USA); Jonathan B. Orens (Johns Hopkins University, Baltimore, MD, USA); Scott M. Palmer (Duke University, Durham, NC, USA); Greg I. Snell (Alfred Hospital, Melbourne, Australia); Marc Stern (Hôpital Foch Suresnes, Paris, France); Sean M. Studer (New York University, Woodhull Medical Center, New York, NY, USA); Stuart C. Sweet (Washington University, St. Louis, MO, USA); Henry D. Tazelaar (Mayo Clinic, Scottsdale, AZ, USA); Timothy P. Whelan 
(Medical University of South Carolina, Charleston, SC, USA); David S. Wilkes (Indiana University School of Medicine, Indianapolis, IN, USA); Trevor J. Williams (University of Melbourne, Melbourne, Australia); Martin R. Zamora (University of Colorado, Denver, CO, USA).

Methodologists: Jan Brozek (McMaster University, Hamilton, ON, Canada) and Kevin C. Wilson (Boston University Medical Center, Boston, MA, USA).

\section{References}

1 Burke CM, Theodore J, Dawkins KD, et al. Post-transplant obliterative bronchiolitis and other late lung sequelae in human heart-lung transplant recipients. Chest 1984; 86: 824-829.

2 Christie JD, Edwards LB, Aurora P, et al. The registry of the International Society for Heart and Lung Transplantation: twenty-sixth official adult lung and heart-lung transplantation report - 2009. J Heart Lung Transplant 2009; 28: 1031-1049.

3 The International Society for Heart and Lung Transplantation. www.ishlt.org

4 Cooper JD, Billingham M, Egan T, et al. A working formulation for the standardization of nomenclature and for clinical staging of chronic dysfunction in lung allografts. International Society for Heart and Lung Transplantation. J Heart Lung Transplant 1993; 12: 713-716.

5 Estenne M, Maurer JR, Boehler A, et al. Bronchiolitis obliterans syndrome 2001: an update of the diagnostic criteria. I Heart Lung Transplant 2002; 21: 297-310.

6 Bando K, Paradis IL, Similo S, et al. Obliterative bronchiolitis after lung and heart-lung transplantation. An analysis of risk factors and management. $J$ Thorac Cardiovasc Surg 1995; 110: 4-13.

7 Reichenspurner H, Girgis RE, Robbins RC, et al. Stanford experience with obliterative bronchiolitis after lung and heart-lung transplantation. Ann Thorac Surg 1996; 62: 1467-1472.

8 Kramer MR, Stoehr C, Whang JL, et al. The diagnosis of obliterative bronchiolitis after heart-lung and lung transplantation: low yield of transbronchial lung biopsy. J Heart Lung Transplant 1993; 12: 675-681.

9 Pomerance A, Madden B, Burke MM, et al. Transbronchial biopsy in heart and lung transplantation: clinicopathologic correlations. J Heart Lung Transplant 1995; 14: 761-773.

10 Chamberlain D, Maurer J, Chaparro C, et al. Evaluation of transbronchial lung biopsy specimens in the diagnosis of bronchiolitis obliterans after lung transplantation. J Heart Lung Transplant 1994; 13: 963-971.

11 Burton CM, Iversen M, Carlsen J, et al. Acute cellular rejection is a risk factor for bronchiolitis obliterans syndrome independent of post-transplant baseline FEV1. J Heart Lung Transplant 2009; 28: 888-893.

12 Schulman LL, Weinberg AD, McGregor C, et al. Mismatches at the HLA-DR and HLA-B loci are risk factors for acute rejection after lung transplantation. Am J Respir Crit Care Med 1998; 157: 1833-1837.

13 Burlingham WJ, Love RB, Jankowska-Gan E, et al. IL-17-dependent cellular immunity to collagen type V predisposes to obliterative bronchiolitis in human lung transplants. J Clin Invest 2007; 117: 3498-3506.

14 Belperio JA, Weigt S, Fishbein MC, et al. Chronic lung allograft rejection: mechanisms and therapy. Proc Am Thorac Soc 2009; 6: 108-121.

15 Weigt SS, Wallace WD, Derhovanessian A, et al. Chronic allograft rejection: epidemiology, diagnosis, pathogenesis, and treatment. Semin Respir Crit Care Med 2010; 31: 189-207.

16 Verleden GM, Vos R, De Vleeschauwer SI, et al. Obliterative bronchiolitis following lung transplantation: from old to new concepts? Transpl Int 2009; 22: 771-779.

17 Schünemann HJ, Jaeschke R, Cook DJ, et al. An official ATS statement: grading the quality of evidence and strength of recommendations in ATS guidelines and recommendations. Am J Respir Crit Care Med 2006; 174: 605-614.

18 Sato M, Waddell TK, Wagnetz U, et al. Restrictive allograft syndrome (RAS): a novel form of chronic lung allograft dysfunction. J Heart Lung Transplant 2011; 30: 735-742.

19 Lama VN, Murray S, Lonigro RJ, et al. Course of FEV1 after onset of bronchiolitis obliterans syndrome in lung transplant recipients. Am J Respir Crit Care Med 2007; 175: 1192-1198.

20 Woodrow JP, Shlobin OA, Barnett SD, et al. Comparison of bronchiolitis obliterans syndrome to other forms of chronic lung allograft dysfunction after lung transplantation. J Heart Lung Transplant 2010; 29: 1159-1164.

21 Jackson CH, Sharples LD, McNeil K, et al. Acute and chronic onset of bronchiolitis obliterans syndrome (BOS): are they different entities? J Heart Lung Transplant 2002; 21: 658-666.

22 Brugière $\mathrm{O}$, Pessione $\mathrm{F}$, Thabut $\mathrm{G}$, et al. Bronchiolitis obliterans syndrome after single-lung transplantation: impact of time to onset on functional pattern and survival. Chest 2002; 121: 1883-1889.

23 Burton CM, Carlsen J, Mortensen J, et al. Long-term survival after lung transplantation depends on development and severity of bronchiolitis obliterans syndrome. J Heart Lung Transplant 2007; 26: 681-686.

24 Nathan SD, Ross DJ, Belman MJ, et al. Bronchiolitis obliterans in single-lung transplant recipients. Chest 1995; 107: 967-972.

25 Vos R, Vanaudenaerde BM, Ottevaere A, et al. Long-term azithromycin therapy for bronchiolitis obliterans syndrome: divide and conquer? J Heart Lung Transplant 2010; 29: 1358-1368.

26 Gottlieb J, Szangolies J, Koehnlein T, et al. Long-term azithromycin for bronchiolitis obliterans syndrome after lung transplantation. Transplantation 2008; 85: 36-41.

27 Vos R, Vanaudenaerde BM, Verleden SE, et al. A randomised controlled trial of azithromycin to prevent bronchiolitis obliterans syndrome after lung transplantation. Eur Respir J 2011; 37: 164-172.

28 Stewart S, Fishbein MC, Snell GI, et al. Revision of the 1996 working formulation for the standardization of nomenclature in the diagnosis of lung rejection. J Heart Lung Transplant 2007; 26: 1229-1242.

29 Keller CA, Cagle PT, Brown RW, et al. Bronchiolitis obliterans in recipients of single, double, and heart-lung transplantation. Chest 1995; 107: 973-980.

30 Girgis RE, Tu I, Berry GJ, et al. Risk factors for the development of obliterative bronchiolitis after lung transplantation. J Heart Lung Transplant 1996; 15: 1200-1208.

31 Kroshus TJ, Kshettry VR, Savik K, et al. Risk factors for the development of bronchiolitis obliterans syndrome after lung transplantation. J Thorac Cardiovasc Surg 1997; 114: 195-202.

32 Heng D, Sharples LD, McNeil K, et al. Bronchiolitis obliterans syndrome: incidence, natural history, prognosis, and risk factors. J Heart Lung Transplant 1998; 17: 1255-1263. 
33 Husain AN, Siddiqui MT, Holmes EW, et al. Analysis of risk factors for the development of bronchiolitis obliterans syndrome. Am J Respir Crit Care Med 1999; 159: 829-833.

34 El-Gamel A, Sim E, Hasleton P, et al. Transforming growth factor beta (TGF- $\beta$ ) and obliterative bronchilitis following pulmonary transplantation. J Heart Lung Transplant 1999; 18: 828-837.

35 Sharples LD, McNeil K, Stewart S, et al. Risk factors for bronchiolitis obliterans: a systematic review of recent publications. J Heart Lung Transplant 2002; 21: 271-281.

36 Burton CM, Iversen M, Scheike T, et al. Is lymphocytic bronchiolitis a marker of acute rejection? An analysis of 2,697 transbronchial biopsies after lung transplantation. J Heart Lung Transplant 2008; 27: 1128-1134.

37 Glanville AR, Aboyoun CL, Havryk A, et al. Severity of lymphocytic bronchiolitis predicts long-term outcome after lung transplantation. Am J Respir Crit Care Med 2008; 177: 1033-1040.

38 Ross DJ, Marchevsky A, Kramer M, et al. "Refractoriness" of airflow obstruction associated with isolated lymphocytic bronchiolitis/bronchitis in pulmonary allografts. J Heart Lung Transplant 1997; 16: 832-838.

39 Guilinger RA, Paradis IL, Dauber JH, et al. The importance of bronchoscopy with transbronchial biopsy and bronchoalveolar lavage in the management of lung transplant recipients. Am J Respir Crit Care Med 1995; 152: 2037-2043.

40 Levine SM, Transplant/Immunology Network of the American College of Chest Physicians. A survey of clinical practice of lung transplantation in North America. Chest 2004; 125: 1224-1238.

41 Hopkins PM, Aboyoun CL, Chhajed PN, et al. Association of minimal rejection in lung transplant recipients with obliterative bronchiolitis. Am J Respir Crit Care Med 2004; 170: 1022-1026.

42 Hachem RR, Khalifah AP, Chakinala MM, et al. The significance of a single episode of minimal acute rejection after lung transplantation. Transplantation 2005; 80: 1406-1413.

43 Khalifah AP, Hachem RR, Chakinala MM, et al. Minimal acute rejection after lung transplantation: a risk for bronchiolitis obliterans syndrome. Am J Transplant 2005; 5: 2022-2030.

44 Palmer SM, Davis RD, Hadjiliadis D, et al. Development of an antibody specific to major histocompatibility antigens detectable by flow cytometry after lung transplant is associated with bronchiolitis obliterans syndrome. Transplantation 2002; 74: 799-804.

45 Lau CL, Palmer SM, Posther KE, et al. Influence of panel-reactive antibodies on posttransplant outcomes in lung transplant recipients. Ann Thorac Surg 2000; 69: 1520-1524.

46 Girnita AL, Duquesnoy R, Yousem SA, et al. HLA-specific antibodies are risk factors for lymphocytic bronchiolitis and chronic lung allograft dysfunction. Am J Transplant 2005; 5: 131-138.

47 Sundaresan S, Mohanakumar T, Smith MA, et al. HLA-A locus mismatches and development of antibodies to HLA after lung transplantation correlate with the development of bronchiolitis obliterans syndrome. Transplantation 1998; 65: 648-653.

48 Jaramillo A, Smith MA, Phelan D, et al. Development of ELISA-detected anti-HLA antibodies precedes the development of bronchiolitis obliterans syndrome and correlates with progressive decline in pulmonary function after lung transplantation. Transplantation 1999; 67: 1155-1161.

49 Bharat A, Narayanan K, Street T, et al. Early posttransplant inflammation promotes the development of alloimmunity and chronic human lung allograft rejection. Transplantation 2007; 83: 150-158.

50 Bharat A, Kuo E, Steward N, et al. Immunological link between primary graft dysfunction and chronic lung allograft rejection. Ann Thorac Surg 2008; 86: 189-195.

51 Daud SA, Yusen RD, Meyers BF, et al. Impact of immediate primary lung allograft dysfunction on bronchiolitis obliterans syndrome. Am J Respir Crit Care Med 2007; 175: 507-513.

52 Huang HJ, Yusen RD, Meyers BF, et al. Late primary graft dysfunction after lung transplantation and bronchiolitis obliterans syndrome. Am J Transplant 2008; 8: 2454-2462.

53 Botha P, Archer L, Anderson RL, et al. Pseudomonas aeruginosa colonization of the allograft after lung transplantation and the risk of bronchiolitis obliterans syndrome. Transplantation 2008; 85: 771-774.

54 Vos R, Vanaudernaerde BM, De Vleeschauwer SI, et al. De novo or persistent pseudomonal airway colonization after lung transplantation: importance for bronchiolitis obliterans syndrome? Transplantation 2008; 86: 624-625.

55 Gottlieb J, Mattner F, Weissbrodt H, et al. Impact of graft colonization with Gram-negative bacteria after lung transplantation on the development of bronchiolitis obliterans syndrome in recipients with cystic fibrosis. Respir Med 2009; 103: 743-749.

56 Keenan RJ, Lega ME, Dummer JS, et al. Cytomegalovirus serologic status and postoperative infection correlated with risk of developing chronic rejection after pulmonary transplantation. Transplantation 1991; 51: 433-438.

57 Smith MA, Sundaresan S, Mohanakumar T, et al. Effect of development of antibodies to HLA and cytomegalovirus mismatch on lung transplantation survival and development of bronchiolitis syndrome. J Thorac Cardiovasc Surg $1998 ; 116 ; 812-820$.

58 Engelmann I, Welte T, Fühner T, et al. Detection of Epstein-Barr virus DNA in peripheral blood is associated with the development of bronchiolitis obliterans syndrome after lung transplantation. J Clin Virol 2009; 45: 47-53.

59 Kumar D, Erdman D, Keshavjee S, et al. Clinical impact of community-acquired respiratory viruses on bronchiolitis obliterans after lung transplant. Am J Transplant 2005; 5: 2031-2036.

60 Bridges ND, Spray TL, Collins MH, et al. Adenovirus infection in the lung results in graft failure after lung transplantation. J Thorac Cardiovasc Surg 1998; 116: 617-623.

61 Khalifah AP, Hachem RR, Chakinala MM, et al. Respiratory viral infections are a distinct risk for bronchiolitis obliterans syndrome and death. Am J Respir Crit Care Med 2004; 170: 181-187.

62 Billings JL, Hertz MI, Savik K, et al. Respiratory viruses and chronic rejection in lung transplant recipients. J Heart Lung Transplant 2002; 21: 559-566.

63 Vilchez RA, Dauber J, McCurry K, et al. Parainfluenza virus infection in adult lung transplant recipients: an emergent clinical syndrome with implications on allograft function. Am J Transplant 2003; 3: 116-120.

64 Palmer SM Jr, Henshaw NG, Howell DN, et al. Community respiratory viral infection in adult lung transplant recipients. Chest 1998; 113: 944-950.

65 D'Ovidio F, Singer LG, Hadjiliadis D, et al. Prevalence of gastroesophageal reflux in end-stage lung disease candidates for lung transplant. Ann Thorac Surg 2005; 80: 1254-1260.

66 Blondeau K, Mertens V, Vanaudenaerde BA, et al. Gastro-oesophageal reflux and gastric aspiration in lung transplant patients with or without chronic rejection. Eur Respir J 2008; 31: 707-713. 
67 King BJ, Iyer $\mathrm{H}$, Leidi AA, et al. Gastroesophageal reflux in bronchiolitis obliterans syndrome: a new perspective. J Heart Lung Transplant 2009; 28: 870-875.

68 Hadjiliadis D, Duane Davis R, Steele MP, et al. Gastroesophageal reflux disease in lung transplant recipients. Clin Transplant 2003; 17: 363-368.

69 Valentine VG, Gupta MR, Walker JE Jr, et al. Effect of etiology and timing of respiratory tract infections on development of bronchiolitis obliterans syndrome. J Heart Lung Transplant 2009; 28: 163-169.

70 Weigt SS, Elashoff RM, Huang C, et al. Aspergillus colonization of the lung allograft is a risk factor for bronchiolitis obliterans syndrome. Am J Transplant 2009; 9: 1903-1911.

71 Weigt SS, Copeland CA, Derhovanessian A, et al. Colonization with small conidia Aspergillus species is associated with bronchiolitis obliterans syndrome: a two-center validation study. Am J Transplant 2013; 13: 919-927.

72 Gandhi MK, Khanna R. Human cytomegalovirus: clinical aspects, immune regulation, and emerging treatments. Lancet Infect Dis 2004; 4: 725-738.

73 Ibrahim L, Dominguez M, Yacoub M. Primary human adult lung epithelial cells in vitro: response to interferon- $\gamma$ and cytomegalovirus. Immunology 1993; 79: 119-124.

74 Geist LJ, Dai LY. Cytomegalovirus modulates interleukin-6 gene expression. Transplantation 1996; 62: 653-658.

75 Vos R, Vanaudenaerde BM, Dupont LJ, et al. Transient airway colonization is associated with airway inflammation after lung transplantation. Am J Transplant 2007; 7: 1278-1287.

76 Bobadilla JL, Jankowska-Gan E, Xu Q, et al. Reflux-induced collagen type v sensitization: potential mediator of bronchiolitis obliterans syndrome. Chest 2010; 138: 363-370.

77 Saini D, Weber J, Ramachandran S, et al. Alloimmunity-induced autoimmunity as a potential mechanism in the pathogenesis of chronic rejection of human lung allografts. J Heart Lung Transplant 2011; 30: 624-631.

78 Henke JA, Golden JA, Yelin EH, et al. Persistent increase of BAL neutrophils as a predictor of mortality following lung transplant. Chest 1999; 115: 403-409.

79 Meyer KC, Nunley DR, Dauber JH, et al. Neutrophils, unopposed neutrophil elastase, and $\alpha 1$-antiprotease defenses following human lung transplantation. Am J Respir Crit Care Med 2001; 164: 97-102.

80 Reynaud-Gaubert M, Marin V, Thirion X, et al. Upregulation of chemokines in bronchoalveolar lavage fluid as a predictive marker of post-transplant airway obliteration. J Heart Lung Transplant 2002; 21: 721-730.

81 DiGiovine B, Lynch JP 3rd, Martinez FJ, et al. Bronchoalveolar lavage neutrophilia is associated with obliterative bronchiolitis after lung transplantation: role of IL-8. J Immunol 1996; 157: 4194-4202.

82 Neurohr C, Huppmann P, Leuchte H, et al. Human herpesvirus 6 in bronchoalveolar lavage fluid after lung transplantation: a risk factor for bronchiolitis obliterans syndrome? Am J Transplant 2005; 5: 2982-2991.

83 Schloma J, Slebos DJ, Boezen HM, et al. Eosinophilic granulocytes and interleukin-6 level in bronchoalveolar lavage fluid are associated with the development of obliterative bronchiolitis after lung transplantation. Am J Respir Crit Care Med 2000; 162: 2221-2225.

84 Lama VN, Murray S, Mumford JA, et al. Prognostic value of bronchiolitis obliterans syndrome stage 0-p in singlelung transplant recipients. Am J Respir Crit Care Med 2005; 172: 379-383.

85 Hachem RR, Chakinala MM, Yusen RD, et al. The predictive value of bronchiolitis obliterans syndrome stage 0-p. Am J Respir Crit Care Med 2004; 169: 468-472.

86 Nathan SD, Barnett SD, Wohlrab J, et al. Bronchiolitis obliterans syndrome: utility of the new guidelines in single lung transplant recipients. J Heart Lung Transplant 2003; 22: 427-432.

87 Morrish WF, Herman SJ, Weisbrod GL, et al. Bronchiolitis obliterans after lung transplantation: findings at chest radiography and high resolution CT. The Toronto Lung Transplant Group. Radiology 1991; 179: 487-490.

88 Kundu S, Herman SJ, Larhs A, et al. Correlation of chest radiographic findings with biopsy-proven acute lung rejection. J Thorac Imaging 1999; 14: 178-184.

89 Collins J. Imaging of the chest after lung transplantation. J Thorac Imaging 2002; 17: 102-112.

90 Leung AN, Fisher K, Valentine V, et al. Bronchiolitis obliterans after lung transplantation: detection using expiratory HRCT. Chest 1998; 113: 365-370.

91 Bankier AA, Van Muylem A, Knoop C, et al. Bronchiolitis obliterans syndrome in heart-lung transplant recipients: diagnosis with expiratory CT. Radiology 2001; 218: 533-539.

92 Lee ES, Gotway MB, Reddy GP, et al. Early bronchiolitis obliterans following lung transplantation: accuracy of expiratory thin-section CT for diagnosis. Radiology 2000; 216: 472-477.

93 Berstad AE, Aaløkken TM, Kolbenstvedt A, et al. Performance of long-term CT monitoring in diagnosing bronchiolitis obliterans after lung transplantation. Eur J Radiol 2006; 58: 124-131.

94 Konen E, Gutierrez C, Chaparro C, et al. Bronchiolitis obliterans syndrome in lung transplant recipients: can thinsection CT findings predict disease before its clinical appearance? Radiology 2004; 231: 467-473.

95 Tepper RS, Reister T. Forced expiratory flows and lung volumes in normal infants. Pediatr Pulmonol 1993; 15 : 357-361.

96 Castile R, Filbrun D, Flucke R, et al. Adult-type pulmonary function tests in infants without respiratory disease. Pediatr Pulmonol 2000; 30: 215-227.

97 Siegel MJ, Bhalla S, Gutierrez FR, et al. Post-lung transplantation bronchiolitis obliterans syndrome: usefulness of expiratory thin-section CT for diagnosis. Radiology 2001; 220: 455-462.

98 Buttgereit F, da Silva JA, Boers M, et al. Standardised nomenclature for glucocorticoid dosages and glucocorticoid treatment regimens: current questions and tentative answers in rheumatology. Ann Rheum Dis 2002; 61: 718-722.

99 Ross DJ, Lewis MI, Kramer M, et al. FK 506 "rescue" immunosuppression for obliterative bronchiolitis after lung transplantation. Chest 1997; 112: 1175-1179.

100 Borro JM, Bravo C, Solé A, et al. Conversion from cyclosporine to tacrolimus stabilizes the course of lung function in lung transplant recipients with bronchiolitis obliterans syndrome. Transplant Proc 2007; 39: 2416-2419.

101 Knoop C, Antoine M, Vachiéry JL, et al. FK 506 rescue therapy for irreversible airway rejection in heart-lung transplant recipients: report on five cases. Transplant Proc 1994; 26: 3240-3241.

102 Reichenspurner H, Meiser BM, Kur F, et al. First experience with FK 506 for treatment of chronic pulmonary rejection. Transplant Proc 1995; 27: 2009.

103 Kesten S, Chaparro C, Scavuzzo M, et al. Tacrolimus as rescue therapy for bronchiolitis obliterans syndrome. J Heart Lung Transplant 1997; 16: 905-912. 
104 Mentzer RM Jr, Jahania MS, Lasley RD. Tacrolimus as a rescue immunosuppressant after heart and lung transplantation. The U.S. Multicenter FK506 Study Group. Transplantation 1998; 65: 109-113.

105 Revell MP, Lewis ME, Llewellyn-Jones CG, et al. Conservation of small-airway function by tacrolimus/cyclosporine conversion in the management of bronchiolitis obliterans following lung transplantation. J Heart Lung Transplant 2000; 19: 1219-1223.

106 Fieguth HG, Krueger S, Wiedenmann DE, et al. Tacrolimus for treatment of bronchiolitis obliterans syndrome after unilateral and bilateral lung transplantation. Transplant Proc 2002; 34: 1884.

107 Cairn J, Yek T, Banner NR, et al. Time-related changes in pulmonary function after conversion to tacrolimus in bronchiolitis obliterans syndrome. J Heart Lung Transplant 2003; 22: 50-57.

108 Sarahrudi K, Estenne M, Corris P, et al. International experience with conversion from cyclosporine to tacrolimus for acute and chronic lung allograft rejection. J Thorac Cardiovasc Surg 2004; 127: 1126-1132.

109 Webster A, Woodroffe RC, Taylor RS, et al. Tacrolimus versus cyclosporin as primary immunosuppression for kidney transplant recipients. Cochrane Database Syst Rev 2005; 4: CD003961.

110 Gerhardt SG, McDyer JF, Girgis RE, et al. Maintenance azithromycin therapy for bronchiolitis obliterans syndrome: results of a pilot study. Am J Respir Crit Care Med 2003; 168: 121-125.

111 Verleden GM, Dupont LJ. Azithromycin therapy for patients with bronchiolitis obliterans syndrome after lung transplantation. Transplantation 2004; 77: 1465-1467.

112 Yates B, Murphy DM, Forrest IA, et al. Azithromycin reverses airflow obstruction in established bronchiolitis obliterans syndrome. Am J Respir Crit Care Med 2005; 172: 772-775.

113 Shitrit D, Bendayan D, Gidon S, et al. Long-term azithromycin use for treatment of bronchiolitis obliterans syndrome in lung transplant recipients. J Heart Lung Transplant 2005; 24: 1440-1443.

114 Verleden GM, Vanaudenaerde BM, Dupont LJ, et al. Azithromycin reduces airway neutrophilia and interleukin-8 in patients with bronchiolitis obliterans syndrome. Am J Respir Crit Care Med 2006; 174: 566-570.

115 Porhownik NR, Batobara W, Kepron W, et al. Effect of maintenance azithromycin on established bronchiolitis obliterans syndrome in lung transplant patients. Can Respir J 2008; 15: 199-202.

116 Jain R, Hachem RR, Morrell MR, et al. Azithromycin is associated with increased survival in lung transplant recipients with bronchiolitis obliterans syndrome. J Heart Lung Transplant 2010; 29: 531-537.

117 Panpanich R, Lerttrakarnnon P, Laopaiboon M. Azithromycin for acute lower respiratory tract infections. Cochrane Database Syst Rev 2008; 1: CD001954.

118 Svanström H, Pasternak B, Hviid A. Use of azithromycin and death from cardiovascular causes. N Engl J Med 2013; 368: 1704-1712.

119 US Food and Drug Administration. FDA Drug Safety Communication: Azithromycin (Zithromax or Zmax) and the risk of potentially fatal heart rhythms. www.fda.gov/drugs/drugsafety/ucm $341822 . h t m$ Date last accessed: September 11, 2013. Date last updated: March 18, 2013.

120 Albert RK, Connett J, Bailey WC, et al. Azithromycin for prevention of exacerbations of COPD. N Engl J Med 2011; 365: 689-698.

121 Young LR, Hadjiliadis D, Davis RD, et al. Lung transplantation exacerbates gastroesophageal reflux disease. Chest 2003; 124: 1689-1693.

122 Sweet MP, Patti MG, Leard LE, et al. Gastroesophageal reflux in patients with idiopathic pulmonary fibrosis referred for lung transplantation. J Thorac Cardiovasc Surg 2007; 133: 1078-1084.

123 Linden PA, Gilbert RJ, Yeap BY, et al. Laparoscopic fundoplication in patients with end-stage lung disease awaiting transplantation. J Thorac Cardiovasc Surg 2006; 131: 438-446.

124 Gasper WJ, Sweet MP, Hoopes C, et al. Antireflux surgery for patients with end-stage lung disease before and after lung transplantation. Surg Endosc 2008; 22: 495-500.

125 Benden C, Aurora P, Curry J, et al. High prevalence of gastroesophageal reflux disease in lung transplant recipients. Pediatr Pulmonol 2005; 40: 68-71.

126 O'Halloran EK, Reynolds JD, Lau CL, et al. Laparoscopic Nissen fundoplication for treating reflux in lung transplant recipients. J Gastrointest Surg 2004; 8: 132-137.

127 Davis RD Jr, Lau CL, Eubanks S, et al. Improved lung allograft function after fundoplication in patients with gastroesophageal reflux disease undergoing lung transplantation. J Thorac Cardiovasc Surg 2003; 125: 533-542.

128 Cantu E 3rd, Appel JZ 3rd, Hartwig MG, et al. J. Maxwell Chamberlain Memorial Paper. Early fundoplication prevents chronic allograft dysfunction in patients with gastroesophageal reflux disease. Ann Thorac Surg 2004; 78: $1142-1151$.

129 Davis CS, Gagermeier J, Dilling D, et al. A review of the potential applications and controversies of non-invasive testing for biomarkers of aspiration in the lung transplant population. Clin Transplant 2010; 24: E54-E61.

130 D'Ovidio F, Mura M, Tsang M, et al. Bile acid aspiration and the development of bronchiolitis obliterans after lung transplantation. J Thorac Cardiovasc Surg 2005; 129: 1144-1152.

131 Hopkins PM, Kermeen F, Duhig E, et al. Oil red O stain of alveolar macrophages is an effective screening test for gastroesophageal reflux disease in lung transplant recipients. J Heart Lung Transplant 2010; 29: 859-864.

132 Lau CL, Palmer SM, Howell DN, et al. Laparoscopic antireflux surgery in the lung transplant population. Surg Endosc 2002; 16: 1674-1678.

133 Zheng C, Kane TD, Kurland G, et al. Feasibility of laparoscopic Nissen fundoplication after pediatric lung or heartlung transplantation: should this be the standard? Surg Endosc 2011; 25: 249-254.

134 Burton PR, Button B, Brown W, et al. Medium-term outcome of fundoplication after lung transplantation. Dis Esophagus 2009; 22: 642-648.

135 Fisichella PM, Davis CS, Gagermeier J, et al. Laparoscopic antireflux surgery for gastroesophageal reflux disease after lung transplantation. J Surg Res 2011; 170: e279-e286.

136 Hartwig MG, Anderson DJ, Onaitis MW, et al. Fundoplication after lung transplantation prevents the allograft dysfunction associated with reflux. Ann Thorac Surg 2011; 92: 462-468.

137 Hoppo T, Jarido V, Pennathur A, et al. Antireflux surgery preserves lung function in patients with gastroesophageal reflux disease and end-stage lung disease before and after lung transplantation. Arch Surg 2011; 146: 1041-1047.

138 Fisichella PM, Davis CS, Lundberg PW, et al. The protective role of laparoscopic antireflux surgery against aspiration of pepsin after lung transplantation. Surgery 2011; 150: 598-606. 
139 Wang Z, Zheng Q, Jin Z. Meta-analysis of robot-assisted versus conventional laparoscopic Nissen fundoplication for gastro-oesophageal reflux disease. ANZ J Surg 2012; 82: 112-117.

140 Khatri K, Sajid MS, Brodrick R, et al. Laparoscopic Nissen fundoplication with or without short gastric vessel division: a meta-analysis. Surg Endosc 2012; 26: 970-978.

141 Brugière $\mathrm{O}$, Thabut $\mathrm{G}$, Castier $\mathrm{Y}$, et al. Lung retransplantation for bronchiolitis obliterans syndrome: long-term follow-up in a series of 15 recipients. Chest 2003; 123: 1832-1837.

142 Strueber M, Fischer S, Gottlieb J, et al. Long-term outcome after pulmonary retransplantation. J Thorac Cardiovasc Surg 2006; 132: 407-412.

143 Aigner C, Jaksch P, Taghavi S, et al. Pulmonary retransplantation: is it worth the effort? A long-term analysis of 46 cases. J Heart Lung Transplant 2008; 27: 60-65.

144 Osaki S, Maloney JD, Meyer KC, et al. Redo lung transplantation for acute and chronic lung allograft failure: longterm follow-up in a single center. Eur J Cardiothorac Surg 2008; 34: 1191-1197.

145 Novick RJ, Stitt L, Schäfers HJ, et al. Pulmonary retransplantation: does the indication for operation influence postoperative lung function? J Thorac Cardiovasc Surg 1996; 112: 1504-1513.

146 Novick RJ, Stitt LW, Al-Kattan K, et al. Pulmonary retransplantation: predictors of graft function and survival in 230 patients. Pulmonary Retransplant Registry. Ann Thorac Surg 1998; 65: 227-234.

147 Kawut SM, Lederer DJ, Keshavjee S, et al. Outcomes after lung retransplantation in the modern era. Am J Respir Crit Care Med 2008; 177: 114-120.

148 Keshavjee S. Retransplantation of the lung comes of age. J Thorac Cardiovasc Surg 2006; 132: 226-228.

149 Sato M, Ohmori-Matsuda K, Saito T, et al. Time-dependent changes in the risk of death in pure bronchiolitis obliterans syndrome (BOS). J Heart Lung Transplant 2013; 32: 484-491.

150 Vanaudenaerde BM, De Vleeschauwer SI, Vos R, et al. The role of the IL23/IL17 axis in bronchiolitis obliterans syndrome after lung transplantation. Am J Transplant 2008; 8: 1911-1920.

151 Serody JS, Hill GR. The IL-17 differentiation pathway and its role in transplant outcome. Biol Blood Marrow Transplant 2012; 18: S56-S61.

152 Shilling RA, Wilkes DS. Role of Th17 cells and IL-17 in lung transplant rejection. Semin Immunopathol 2011; 33: 129-134.

153 Vanaudenaerde BM, Verleden SE, Vos R, et al. Innate and adaptive interleukin-17-producing lymphocytes in chronic inflammatory lung disorders. Am J Respir Crit Care Med 2011; 183: 977-986.

154 Afzali B, Lombardi G, Lechler RI, et al. The role of T helper 17 (Th17) and regulatory T cells (Treg) in human organ transplantation and autoimmune disease. Clin Exp Immunol 2007; 148: 32-46.

155 Fan L, Benson HL, Vittal R, et al. Neutralizing IL-17 prevents obliterative bronchiolitis in murine orthotopic lung transplantation. Am J Transplant 2011; 11: 911-922.

156 Vittal R, Fan L, Greenspan DS, et al. IL-17 induces type V collagen overexpression and EMT via TGF- $\beta$ dependent pathways in obliterative bronchiolitis. Am J Physiol Lung Cell Mol Physiol 2013; 304: L401-L414.

157 Bettelli E, Carrier Y, Gao W, et al. Reciprocal developmental pathways for the generation of pathogenic effector TH17 and regulatory T cells. Nature 2006; 441: 235-238.

158 Neujahr DC, Larsen CP. Regulatory T cells in lung transplantation - an emerging concept. Semin Immunopathol 2011; 33: 117-127.

159 Tiriveedhi V, Takenaka M, Ramachandran S, et al. T regulatory cells play a significant role in modulating MHC class I antibody-induced obliterative airway disease. Am J Transplant 2012; 12: 2663-2674.

160 Shi Q, Cao H, Liu J, et al. $\mathrm{CD}_{4}^{+}$Foxp $^{+}$regulatory T cells induced by TGF- $\beta$, IL-2 and all-trans retinoic acid attenuate obliterative bronchiolitis in rat trachea transplantation. Int Immunopharmacol 2011; 11: 1887-1894.

161 Braun RK, Molitor-Dart M, Wigfield C, et al. Transfer of tolerance to collagen type V suppresses T-helper-cell-17 lymphocyte-mediated acute lung transplant rejection. Transplantation 2009; 88: 1341-1348.

162 Griffin DO, Rothstein TL. Human "orchestrator" CD11b ${ }^{+}$B1 cells spontaneously secrete interleukin-10 and regulate T-cell activity. Mol Med 2012; 18: 1003-1008.

163 Li W, Bribriesco AC, Nava RG, et al. Lung transplant acceptance is facilitated by early events in the graft and is associated with lymphoid neogenesis. Mucosal Immunol 2012; 5: 544-554.

164 Ward C, Forrest IA, Murphy DM, et al. Phenotype of airway epithelial cells suggests epithelial to mesenchymal cell transition in clinically stable lung transplant recipients. Thorax 2005; 60: 865-871.

165 Hodge S, Holmes M, Banerjee B, et al. Posttransplant bronchiolitis obliterans syndrome is associated with bronchial epithelial to mesenchymal transition. Am J Transplant 2009; 9: 727-733.

166 Borthwick LA, Parker SM, Brougham KA, et al. Epithelial to mesenchymal transition (EMT) and airway remodelling after human lung transplantation. Thorax 2009; 64: 770-777.

167 Gardner A, Fisher AJ, Richter C, et al. The critical role of TAK1 in accentuated epithelial to mesenchymal transition in obliterative bronchiolitis after lung transplantation. Am J Pathol 2012; 180: 2293-2308.

168 Cypel M, Yeung JC, Hirayama S, et al. Technique for prolonged normothermic ex vivo lung perfusion. J Heart Lung Transplant 2008; 27: 1319-1325.

169 Cypel M, Rubacha M, Yeung J, et al. Normothermic ex vivo perfusion prevents lung injury compared to extended cold preservation for transplantation. Am J Transplant 2009; 9: 2262-2269.

170 Ingemansson R, Eyjolfsson A, Mared L, et al. Clinical transplantation of initially rejected donor lungs after reconditioning ex vivo. Ann Thorac Surg 2009; 87: 255-260.

171 Aigner C, Slama A, Hötzenecker K, et al. Clinical ex vivo lung perfusion - pushing the limits. Am J Transplant 2012; 12: 1839-1847.

172 Zych B, Popov AF, Stavri G, et al. Early outcomes of bilateral sequential single lung transplantation after ex-vivo lung evaluation and reconditioning. J Heart Lung Transplant 2012; 31: 274-281.

173 Fisher AJ, Rutherford RM, Bozzino J, et al. The safety and efficacy of total lymphoid irradiation in progressive bronchiolitis obliterans syndrome after lung transplantation. Am J Transplant 2005; 5: 537-543.

174 Diamond DA, Michalski JM, Lynch JP, et al. Efficacy of total lymphoid irradiation for chronic allograft rejection following bilateral lung transplantation. Int J Radiat Oncol Biol Phys 1998; 41: 795-800.

175 Benden C, Speich R, Hofbauer GF, et al. Extracorporeal photopheresis after lung transplantation: a 10-year singlecenter experience. Transplantation 2008; 86: 1625-1627. 
176 Morrell MR, Despotis GJ, Lublin DM, et al. The efficacy of photopheresis for bronchiolitis obliterans syndrome after lung transplantation. J Heart Lung Transplant 2010; 29: 424-431.

177 Cahill BC, Somerville KT, Crompton JA, et al. Early experience with sirolimus in lung transplant recipients with chronic allograft rejection. J Heart Lung Transplant 2003; 22: 169-176.

178 Reams BD, Musselwhite LW, Zaas DW, et al. Alemtuzumab in the treatment of refractory acute rejection and bronchiolitis obliterans syndrome after human lung transplantation. Am J Transplant 2007; 7: 2802-2808.

179 Shyu S, Dew MA, Pilewski JM, et al. Five-year outcomes with alemtuzumab induction after lung transplantation. J Heart Lung Transplant 2011; 30: 743-754.

180 Cai J, Terasaki PI. Induction immunosuppression improves long-term graft and patient outcome in organ transplantation: an analysis of United Network for Organ Sharing registry data. Transplantation 2010; 90: 1511-1515. 